\title{
Pacific
}

Journal of

Mathematics

\section{ONE LINE COMPLEX KLEINIAN GROUPS}

Waldemar Barrera, Angel Cano and Juan Pablo Navarrete 


\title{
ONE LINE COMPLEX KLEINIAN GROUPS
}

\author{
Waldemar Barrera, Angel CANo And JuAn Pablo Navarrete
}

\begin{abstract}
We give an algebraic description of those subgroups of $\operatorname{PGL}(3, \mathbb{C})$ acting on $\mathbb{P}_{\mathbb{C}}^{2}$ with Kulkarni limit set equal to one complex projective line. Conversely, we prove that the Kulkarni limit set of a group $G \leq \operatorname{PGL}(3, \mathbb{C})$ acting properly and discontinuously on the complement of one line in $\mathbb{P}_{\mathbb{C}}^{2}$ is equal to one or two lines.
\end{abstract}

\section{Introduction}

The Kleinian groups are discrete subgroups of $\operatorname{PSL}(2, \mathbb{C})$ acting on $\mathbb{S}^{2} \cong \mathbb{P}_{\mathbb{C}}^{1}$ in such way that its limit set is not all of $\mathbb{S}^{2}$. They are classified in elementary and nonelementary groups. The elementary groups are those Kleinian groups whose limit set is equal to zero, one or two points, and they are classified (see [Maskit 1988]). The nonelementary groups are those Kleinian groups whose limit set contains more than two points and in this case its limit set is a perfect set.

Our interest relies on the study of complex Kleinian groups. These are discrete subgroups of PGL $(3, \mathbb{C})$ acting properly and discontinuously on some open subset of $\mathbb{P}_{\mathbb{C}}^{2}$. In this setting, there is no standard definition of limit set, however, in [Barrera Vargas et al. 2011] it is proved that under some mild hypothesis on the dynamics of the group, Kulkarni's definition of limit set is an appropriate definition (see Definition 2.1).

In [Cano and Seade 2014] it is proved that the Kulkarni limit set of a complex Kleinian group contains a complex projective line whenever the group is infinite. Moreover, in [Barrera Vargas et al. 2011] it is proved that under some mild hypothesis on the group, the Kulkarni limit set is a union of complex projective lines. The definition of elementary group in this case is that the Kulkarni limit set consists of a finite union of complex projective subspaces (see [Cano et al. 2013]).

An interesting problem consists of classifying all elementary complex Kleinian groups, and one natural step consists of classifying those discrete subgroups of $\operatorname{PGL}(3, \mathbb{C})$ whose Kulkarni limit set consists of one complex projective line. In

Research supported by CONACYT Project Number 176680 and SEP grant P/PIFI-2011-31MSU0098J15.

MSC2010: 37F30, 22E40.

Keywords: complex Kleinian groups, Kulkarni limit set, complex projective plane. 
this paper we prove that the complex Kleinian group $G \leq \operatorname{PGL}(3, \mathbb{C})$ is virtually nilpotent whenever its Kulkarni limit set is equal to one complex projective line. In fact we prove the following:

Theorem 1.1. If $G$ is a subgroup of $\operatorname{PGL}(3, \mathbb{C})$ such that its Kulkarni limit set $\Lambda(G)$ consists of precisely one complex projective line $\ell$, then:

(i) If $G$ contains a loxoparabolic element then $G$ is a finite cyclic extension of order $1,2,3,4$ or 6 of $\mathbb{Z} \oplus \mathbb{Z} \oplus \mathbb{Z}_{n_{0}}$, where $n_{0} \in \mathbb{N}$ is arbitrary. The $\mathbb{Z} \oplus \mathbb{Z}$ is a group of rank two generated by a loxoparabolic element and another element which can be loxoparabolic or parabolic. Also, the $\mathbb{Z}_{n_{0}}$ is a group of complex reflections.

(ii) If $G$ does not contain any loxoparabolic elements and the group $G$ does not contain any element which acts as a parabolic element on the complex line $\Lambda(G)=\ell$, then $G$ is a group of isometries of $\mathbb{C}^{2}$ and it contains a free abelian normal subgroup of finite index and of rank less than or equal to four.

(iii) If $G$ does not contain any loxoparabolic elements but it does contain an element which acts as a parabolic element on the complex line $\Lambda(G)=\ell$, then $G$ does not contain any irrational ellipto-parabolic elements and it is a finite extension of a unipotent subgroup (this subgroup consists of unipotent parabolic maps). Hence it is a finite extension of a group of the form $\mathbb{Z}, \mathbb{Z}^{2}, \mathbb{Z}^{3}, \mathbb{Z}^{4}, \Delta_{k}$ or $\Gamma_{k}$, where

$$
\Delta_{k}=\left\langle A, B, C, D: C, D \text { are central and }[A, B]=C^{k}\right\rangle, \quad k \in \mathbb{N},
$$

and

$$
\Gamma_{k}=\left\langle A, B, C: C \text { is central and }[A, B]=C^{k}\right\rangle, \quad k \in \mathbb{N} .
$$

The outline of the proof of Theorem 1.1 is as follows: Since the group acts properly and discontinuously on the complement of one complex projective line in $\mathbb{P}_{\mathbb{C}}^{2}$, the dynamics of each element in the group are restricted in some way; see Remark 2.4. In fact, the elements in the group are elliptic, parabolic or loxoparabolic according to the classification given in [Navarrete 2008].

If the group contains a loxoparabolic element, then there are restrictions on the group $G$, as shown in Lemma 3.1. The proof of Theorem 1.1(i) follows from the fact that there exists an invariant complex projective line where the action of the group is properly discontinuous except in one point. Hence the group acts as a Euclidean group on this line.

If the group does not contain any loxoparabolic elements, then we consider the following two cases:

If $G$ acts on the limit set $\ell \cong \hat{\mathbb{C}} \cong \mathbb{S}^{2}$ without parabolic elements then $G$ can be considered as a group of Euclidean isometries of $\mathbb{R}^{4}$. 
If some element in $G$ acts on the limit set $\ell$ as parabolic element then the group can be identified with a group of triangular matrices (see Proposition 5.2). The existence of irrational ellipto-parabolic elements in the group is ruled out by Propositions 5.6 and 5.7. Finally, there exists a unipotent subgroup of finite index (see Proposition 5.8.)

We remark that not every finite extension of those nilpotent groups given in Theorem 1.1(ii) and (iii) can occur as a group with Kulkarni limit set equal to one line. Which of them can occur is a more delicate question. However, Theorem 1.1 gives a qualitative description according to the dynamics of the kind of elements contained in the group.

We are not restricting here to the case where the quotient space $\mathbb{P}_{\mathbb{C}}^{2} \backslash \ell$ by the group $G$ is compact. The case where the action of $G \leq \operatorname{PGL}(3, \mathbb{C})$ on $\mathbb{P}_{\mathbb{C}}^{2} \backslash \ell$ is free, properly discontinuous and the quotient is compact is handled in [Fillmore and Scheuneman 1973; Scheuneman 1974; Suwa 1975].

Finally, if $G \leq \operatorname{PGL}(3, \mathbb{C})$ satisfies $\Lambda(G)$ is equal to one line, then $G$ acts properly and discontinuously on the complement of one line in $\mathbb{P}_{\mathbb{C}}^{2}$, so $G$ can be considered as a discrete subgroup of $\operatorname{Aff}\left(\mathbb{C}^{2}\right)$ acting properly and discontinuously on $\mathbb{C}^{2}$. The converse statement is not true as we show in the following:

Theorem 1.2. Let $G \leq \operatorname{PGL}(3, \mathbb{C})$ be an infinite group which acts properly and discontinuously on the complement of the line $\ell \subset \mathbb{P}_{\mathbb{C}}^{2}$.

(i) If $G$ contains a loxoparabolic element then,

- the Kulkarni limit set $\Lambda(G)$ is equal to the union of $\ell$ and another line whenever $G$ contains a cyclic subgroup of finite index generated by a loxoparabolic element, or

- the Kulkarni limit set $\Lambda(G)$ is equal to $\ell$ whenever $G$ contains a finite-index free abelian subgroup of rank two generated by a loxoparabolic element and another element, which can be either loxoparabolic or parabolic.

(ii) If $G$ does not contain any loxoparabolic elements then $\Lambda(G)=\ell$.

\section{Preliminaries}

2.1. Projective geometry. Recall that the complex projective plane $\mathbb{P}_{\mathbb{C}}^{2}$ is defined as

$$
\mathbb{P}_{\mathbb{C}}^{2}:=\left(\mathbb{C}^{3} \backslash\{\mathbf{0}\}\right) / \mathbb{C}^{*},
$$

where $\mathbb{C}^{*}=\mathbb{C} \backslash\{0\}$ acts on $\mathbb{C}^{3} \backslash\{\mathbf{0}\}$ by the usual scalar multiplication. This is a compact connected complex 2-dimensional manifold. Let $[\cdot]: \mathbb{C}^{3} \backslash\{\mathbf{0}\} \rightarrow \mathbb{P}_{\mathbb{C}}^{2}$ be the quotient map. If $\beta=\left\{e_{1}, e_{2}, e_{3}\right\}$ is the standard basis of $\mathbb{C}^{3}$, we write $\left[e_{j}\right]=e_{j}$ and if $\mathbf{z}=\left(z_{1}, z_{2}, z_{3}\right) \in \mathbb{C}^{3} \backslash\{\mathbf{0}\}$ then we write $[\mathbf{z}]=\left[z_{1}: z_{2}: z_{3}\right]$. Also, $\ell \subset \mathbb{P}_{\mathbb{C}}^{2}$ is said to be a complex line if $[\ell]^{-1} \cup\{\boldsymbol{0}\}$ is a complex linear subspace of dimension two. 
Given distinct points $[\mathbf{z}],[\mathbf{w}] \in \mathbb{P}_{\mathbb{C}}^{2}$, there is a unique complex projective line passing through $[\mathbf{z}]$ and $[\mathbf{w}]$. Such a complex projective line is called a line, for short, and it is denoted by $[\mathbf{z}],[\mathbf{w}]$. Consider the action of $\mathbb{C}^{*}$ on $\operatorname{GL}(3, \mathbb{C})$ given by the usual scalar multiplication. Then

$$
\operatorname{PGL}(3, \mathbb{C})=\operatorname{GL}(3, \mathbb{C}) / \mathbb{C}^{*}
$$

is a Lie group. The elements of this Lie group are called projective transformations. Let $[[\cdot]]: \operatorname{GL}(3, \mathbb{C}) \rightarrow \operatorname{PGL}(3, \mathbb{C})$ be the quotient map, $g \in \operatorname{PGL}(3, \mathbb{C})$ and $\mathbf{g} \in \mathrm{GL}(3, \mathbb{C})$. We say that $\mathbf{g}$ is a lift of $g$ if $[[\mathbf{g}]]=g$. One can show that $\operatorname{PGL}(3, \mathbb{C})$ is a Lie group that acts transitively, effectively and by biholomorphisms on $\mathbb{P}_{\mathbb{C}}^{2}$ by $[[\mathbf{g}]]([\mathbf{w}])=[\mathbf{g}(\mathbf{w})]$, where $\mathbf{w} \in \mathbb{C}^{3} \backslash\{\mathbf{0}\}$ and $\mathbf{g} \in \mathrm{GL}(3, \mathbb{C})$.

The Fubini-Study metric on $\mathbb{P}_{\mathbb{C}}^{2}$ is a useful tool in the computation of the Kulkarni limit set of cyclic subgroups of PGL(3, $\mathbb{C}$ ) acting on $\mathbb{P}_{\mathbb{C}}^{2}$ (see [Navarrete 2008]). The Fubini-Study distance $d([\mathbf{z}],[\mathbf{w}])$ between $[\mathbf{z}],[\mathbf{w}] \in \mathbb{P}_{\mathbb{C}}^{2}$ satisfies the equation

$$
\cos ^{2}(d([\mathbf{z}],[\mathbf{w}]))=\frac{\left|z_{1} \bar{w}_{1}+z_{2} \bar{w}_{2}+z_{3} \bar{w}_{3}\right|^{2}}{\left(\left|z_{1}\right|^{2}+\left|z_{2}\right|^{2}+\left|z_{3}\right|^{2}\right)\left(\left|w_{1}\right|^{2}+\left|w_{2}\right|^{2}+\left|w_{3}\right|^{2}\right)} .
$$

We denote by $M_{3 \times 3}(\mathbb{C})$ the space of all $3 \times 3$ matrices with entries in $\mathbb{C}$ equipped with the standard topology. The quotient space

$$
\left(M_{3 \times 3}(\mathbb{C}) \backslash\{\boldsymbol{0}\}\right) / \mathbb{C}^{*}
$$

is called the space of pseudo-projective maps of $\mathbb{P}_{\mathbb{C}}^{2}$ and it is naturally identified with the projective space $\mathbb{P}_{\mathbb{C}}^{8}$. Since $\operatorname{GL}(3, \mathbb{C})$ is an open, dense, $\mathbb{C}^{*}$-invariant set of $M_{3 \times 3}(\mathbb{C}) \backslash\{\boldsymbol{0}\}$, we obtain that the space of pseudo-projective maps of $\mathbb{P}_{\mathbb{C}}^{2}$ is a compactification of $\operatorname{PGL}(3, \mathbb{C})$. As in the case of projective maps, if $\mathbf{s}$ is an element in $M_{3 \times 3}(\mathbb{C}) \backslash\{\mathbf{0}\}$, then $[\mathbf{s}]$ denotes the equivalence class of the matrix $\mathbf{s}$ in the space of pseudo-projective maps of $\mathbb{P}_{\mathbb{C}}^{2}$. Also, we say that $\mathbf{s} \in M_{3 \times 3}(\mathbb{C}) \backslash\{\mathbf{0}\}$ is a lift of the pseudo-projective map $S$ whenever $[\mathbf{s}]=S$.

Let $S$ be an element in $\left(M_{3 \times 3}(\mathbb{C}) \backslash\{\boldsymbol{0}\}\right) / \mathbb{C}^{*}$ and $\mathbf{s}$ a lift to $M_{3 \times 3}(\mathbb{C}) \backslash\{\boldsymbol{0}\}$ of $S$. The matrix $\mathbf{s}$ induces a nonzero linear transformation $s: \mathbb{C}^{3} \rightarrow \mathbb{C}^{3}$, which is not necessarily invertible. Let $\operatorname{Ker} s \subsetneq \mathbb{C}^{3}$ be its kernel and let $\operatorname{Ker} S$ denote its projectivization to $\mathbb{P}_{\mathbb{C}}^{2}$, taking into account that $\operatorname{Ker} S:=\varnothing$ whenever $\operatorname{Ker} s=$ $\{(0,0,0)\}$. We refer to [Cano and Seade 2010] for more details about this subject.

2.2. Complex Kleinian groups. We recall that a point $z \in \hat{\mathbb{C}} \cong \mathbb{S}^{2} \cong \mathbb{P}_{\mathbb{C}}^{1}$ is a limit point of the discrete subgroup $G$ of $\operatorname{PSL}(2, \mathbb{C})$ if it is a cluster point of some orbit $\{g x: g \in G\}$, where $x \in \hat{\mathbb{C}}$. The set $\Lambda(G)=\{z \in \hat{\mathbb{C}}: z$ is a limit point of $G\}$ is called the limit set of $G$ (see [Marden 2007]). 
It is a known fact that the action of $G$ on the complement of the limit set $\hat{\mathbb{C}} \backslash \Lambda(G)$ is properly discontinuous. However, when working on higher dimensions, this is no longer valid. See Example 2.3 below.

Kulkarni considers a very general setting of discrete group actions on a topological space $X$, and the Kulkarni limit set provides a canonical choice of a closed $G$-invariant set in $X$ such that the $G$-action on its complement is properly discontinuous.

Definition 2.1. Let $G \subset \operatorname{PGL}(n+1, \mathbb{C})$ be a subgroup. The set $L_{0}(G)$ is defined as the closure of the points in $\mathbb{P}_{\mathbb{C}}^{n}$ with infinite isotropy group. In other words,

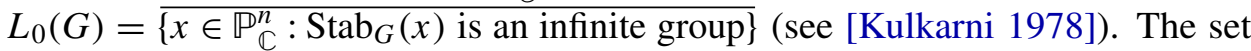
$L_{1}(G)$ is the closure of the set of cluster points of the $G$-orbit of $z$, where $z$ runs over $\mathbb{P}_{\mathbb{C}}^{n} \backslash L_{0}(G)$. Recall that $q$ is a cluster point of the family of sets $\{g(K): g \in G\}$, where $K \subset \mathbb{P}_{\mathbb{C}}^{n}$ is a nonempty set, if there is a sequence $\left(k_{m}\right)_{m \in \mathbb{N}} \subset K$ and a sequence of distinct elements $\left(g_{m}\right)_{m \in \mathbb{N}} \subset G$ such that $g_{m}\left(k_{m}\right) \underset{m \rightarrow \infty}{\longrightarrow} q$. The set $L_{2}(G)$ is defined as the closure of the union of cluster points of $\{g(K): g \in G\}$, where $K$ runs over all the compact sets in $\mathbb{P}_{\mathbb{C}}^{n} \backslash\left(L_{0}(G) \cup L_{1}(G)\right)$. The Kulkarni limit set for $G$ is defined as the $G$-invariant closed set

$$
\Lambda(G)=L_{0}(G) \cup L_{1}(G) \cup L_{2}(G) .
$$

The discontinuity region in the sense of Kulkarni of $G$ is defined as

$$
\Omega(G)=\mathbb{P}_{\mathbb{C}}^{n} \backslash \Lambda(G) .
$$

If $\Omega(G) \neq \varnothing$ then we say that $G$ is a complex Kleinian group.

In the case of a cyclic group $\langle g\rangle$, we write $L_{0}(g), L_{1}(g)$, etc. instead of $L_{0}(\langle g\rangle)$, $L_{1}(\langle g\rangle)$, etc.

The following lemma is a useful tool for the computation of Kulkarni limit sets, and we include it here for reader's convenience. See [Navarrete 2008] for a proof.

Lemma 2.2. Let $G$ be a subgroup of $\mathrm{PGL}(3, \mathbb{C})$. If $C$ is a closed set such that for every compact set $K \subset \mathbb{P}_{\mathbb{C}}^{2} \backslash C$, the cluster points of the family of compact sets $\{g(K)\}_{g \in G}$ are contained in $L_{0}(G) \cup L_{1}(G)$, then $L_{2}(G) \subset C$.

Example 2.3. If $g \in \operatorname{PGL}(3, \mathbb{C})$ is induced by the matrix

$$
\mathbf{g}=\left(\begin{array}{lll}
1 & 0 & 1 \\
0 & \lambda & 0 \\
0 & 0 & 1
\end{array}\right), \quad \lambda \in \mathbb{C}^{*},|\lambda|<1,
$$

then:

(i) $L_{0}(g)=\left\{e_{1}, e_{2}\right\}$.

(ii) $g^{-n}(\cdot) \underset{n \rightarrow \infty}{\longrightarrow} e_{2}$ uniformly on compact subsets of $\mathbb{P}_{\mathbb{C}}^{2} \backslash\left(\overleftrightarrow{e_{1}, e_{2}} \cup \overleftrightarrow{e_{1}, e_{3}}\right)$. 
(iii) $g^{n}(\cdot) \underset{n \rightarrow \infty}{\longrightarrow} e_{1}$ uniformly on compact subsets of $\mathbb{P}_{\mathbb{C}}^{2} \backslash\left(\overleftrightarrow{e_{1}, e_{2}} \cup \overleftrightarrow{e_{1}, e_{3}}\right)$.

(iv) $L_{1}(g)=\left\{e_{1}, e_{2}\right\}$.

(v) $L_{2}(g)=\overleftrightarrow{e_{1}, e_{2}} \cup \overleftrightarrow{e_{1}, e_{3}}$.

(vi) The action of the cyclic group generated by $g$ on $\mathbb{P}_{\mathbb{C}}^{2} \backslash\left\{e_{1}, e_{2}\right\}$ is not properly discontinuous.

(vii) The cyclic group generated by $g$ acts properly and discontinuously on $\mathbb{P}_{\mathbb{C}}^{2} \backslash \overleftrightarrow{e_{1}, e_{2}}$. Proof.

(i) The proof follows straightforwardly from the fact that $\left\{\left(z_{1}, 0,0\right): z_{1} \in \mathbb{C}\right\}$ and $\left\{\left(0, z_{2}, 0\right): z_{2} \in \mathbb{C}\right\}$ are the only possible eigenspaces for each matrix of the form

$$
\mathbf{g}^{n}=\left(\begin{array}{lll}
1 & 0 & n \\
0 & \lambda^{n} & 0 \\
0 & 0 & 1
\end{array}\right), \quad n \in \mathbb{Z} \backslash\{0\} .
$$

(ii) If $K$ is a compact subset of $\mathbb{P}_{\mathbb{C}}^{2} \backslash\left(\overleftrightarrow{e_{1}, e_{2}} \cup \overleftrightarrow{e_{1}, e_{3}}\right)$, then every point in $K$ can be written as $[\mathbf{z}]=\left[z_{1}: z_{2}: z_{3}\right]$, where $\left|z_{1}\right|^{2}+\left|z_{2}\right|^{2}+\left|z_{3}\right|^{2}=1,\left|z_{2}\right| \geq \epsilon$ and $\left|z_{3}\right| \geq \epsilon$ for some fixed $\epsilon>0$. If $d\left(g^{-n}[\mathbf{z}], e_{2}\right)$ denotes the Fubini-Study distance between $g^{-n}[\mathbf{z}]$ and $e_{2}$, then

$$
\begin{aligned}
\cos ^{2}\left(d\left(g^{-n}([\mathbf{z}]), e_{2}\right)\right) & =\frac{\left|\lambda^{-n} z_{2}\right|^{2}}{\left|z_{1}-n z_{3}\right|^{2}+\left|\lambda^{-n} z_{2}\right|^{2}+\left|z_{3}\right|^{2}} \\
& \geq \frac{\left|\lambda^{-n} z_{2}\right|^{2}}{(n \epsilon+1)^{2}+\left|\lambda^{-n} z_{2}\right|^{2}+1} \\
& \geq \frac{|\lambda|^{-2 n} \epsilon^{2}}{(n \epsilon+1)^{2}+|\lambda|^{-2 n} \epsilon^{2}+1},
\end{aligned}
$$

and the last expression goes to 1 as $n \rightarrow \infty$. Therefore, $g^{-n}(\cdot) \underset{n \rightarrow \infty}{\longrightarrow} e_{2}$ uniformly on compact subsets of $\mathbb{P}_{\mathbb{C}}^{2} \backslash\left(\overleftrightarrow{e_{1}, e_{2}} \cup \overleftrightarrow{e_{1}, e_{3}}\right)$.

(iii) The proof is analogous to (ii)

(iv) Since $g$ acts on the invariant line $\overleftrightarrow{e_{1}, e_{2}}$ as a loxodromic element of PGL $(2, \mathbb{C})$ with fixed points $e_{1}$ and $e_{2}$, the orbits of the points in $\overleftrightarrow{e_{1}, e_{2}} \backslash\left\{e_{1}, e_{2}\right\}$ accumulate at $e_{1}$ and $e_{2}$.

Also, $g$ acts on the invariant line $\overleftrightarrow{e_{1}, e_{3}}$ as a parabolic element of PGL $(2, \mathbb{C})$ with fixed point at $e_{1}$, so the orbits of the points in $\overleftrightarrow{e_{1}, e_{3}} \backslash\left\{e_{1}\right\}$ accumulate at $e_{1}$.

Finally, by (ii) and (iii), the set of cluster points of the orbits of points in $\mathbb{P}_{\mathbb{C}}^{2} \backslash\left(\overleftrightarrow{e_{1}, e_{2}} \cup \overleftrightarrow{e_{1}, e_{3}}\right)$ is equal to $\left\{e_{1}, e_{2}\right\}$

(v) By (ii) and (iii), for every compact set $K \subset \mathbb{P}_{\mathbb{C}}^{2} \backslash\left(\overleftrightarrow{e_{1}, e_{2}} \cup \overleftrightarrow{e_{1}, e_{3}}\right)$, the cluster points of the family of compact sets $\left\{g^{n}(K)\right\}_{n \in \mathbb{Z}}$ is equal to $\left\{e_{1}, e_{2}\right\} \subset L_{0}(g) \cup L_{1}(g)$. It follows, by Lemma 2.2, that $L_{2}(g) \subset \overleftrightarrow{e_{1}, e_{2}} \cup \overleftrightarrow{e_{1}, e_{3}}$. 
Conversely, for every $z \in \mathbb{C}^{*}$, the compact set

$$
K_{z}=\left\{\left[z+\lambda^{n}-\frac{1}{n}: 1:-\frac{z}{n}\right] \mid n \in \mathbb{N}\right\} \cup\{[z: 1: 0]\}
$$

is contained in $\mathbb{P}_{\mathbb{C}}^{2} \backslash\left\{e_{1}, e_{2}\right\}=\mathbb{P}_{\mathbb{C}}^{2} \backslash\left(L_{0}(g) \cup L_{1}(G)\right)$. Since

$$
\begin{aligned}
g^{n}\left(\left[z+\lambda^{n}-\frac{1}{n}: 1:-\frac{z}{n}\right]\right) & =\left[\lambda^{n}-\frac{1}{n}: \lambda^{n}:-\frac{z}{n}\right] \\
& =\left[n \lambda^{n}-1: n \lambda^{n}:-z\right] \underset{n \rightarrow \infty}{\longrightarrow}[1: 0: z],
\end{aligned}
$$

it follows that $\overleftrightarrow{e_{1}, e_{3}} \subset L_{2}(g)$

Similarly, for every $z \in \mathbb{C}^{*}$, consider the compact set

$$
\left\{\left[n: n z:-\lambda^{-n}\right] \mid n \in \mathbb{N}\right\} \cup\left\{e_{3}\right\} \subset \mathbb{P}_{\mathbb{C}}^{2} \backslash\left(L_{0}(g) \cup L_{1}(G)\right) .
$$

Since $g^{-n}\left(\left[n: n z:-\lambda^{-n}\right]\right)=\left[\lambda^{n}+1: z:-1 / n\right] \underset{n \rightarrow \infty}{\longrightarrow}[1: z: 0]$, it follows that $\overleftrightarrow{e_{1}, e_{2}} \subset L_{2}(g)$.

(vi) If $K_{z}$ is as in the proof of (v), then $g^{n}\left(K_{z}\right)$ intersects any compact neighborhood of $[1: 0: z]$ for infinitely many values of $n \in \mathbb{Z}$.

(vii) The set $\mathbb{P}_{\mathbb{C}}^{2} \backslash \overleftrightarrow{e_{1}, e_{2}}$ is naturally identified with $\mathbb{C}^{2}$ by the map $\left[z_{1}: z_{2}: 1\right] \mapsto$ $\left(z_{1}, z_{2}\right)$, and the action is now $g\left(z_{1}, z_{2}\right)=\left(z_{1}+1, \lambda z_{2}\right)$.

If for some fixed $R>0,\left(z_{1}, z_{2}\right) \in \mathbb{C}^{2}$ satisfies

$$
\left\|\left(z_{1}, z_{2}\right)\right\|_{1}=\left|z_{1}\right|+\left|z_{2}\right| \leq R,
$$

and

$$
\left\|g^{n}\left(z_{1}, z_{2}\right)\right\|_{1}=\left|z_{1}+n\right|+\left|\lambda^{n} z_{2}\right| \leq R,
$$

then $|n| \leq\left|z_{1}+n\right|+\left|z_{1}\right| \leq 2 R$. Hence (2) and (3) are satisfied for finitely many values of $n \in \mathbb{Z}$. Therefore the cyclic group $\langle g\rangle$ acts properly discontinuously on $\mathbb{P}_{\mathbb{C}}^{2} \backslash \overleftrightarrow{e_{1}, e_{2}}$.

By conformal properties, we have that the Kulkarni limit set of a discrete subgroup of PGL $(2, \mathbb{C})$ acting on $\hat{\mathbb{C}}$ agrees with its classical limit set. In fact, $L_{0}=L_{1}=L_{2}=\Lambda$ in that case. However, when working in higher dimensional projective geometry, the sets $L_{0}, L_{1}$ and $L_{2}$ can be quite different amongst themselves. Moreover, the set $\Omega(G)$ is not always the maximal open subset where the action is properly discontinuous, as illustrated in Example 2.3(vii). Nevertheless, when $G$ acts on $\mathbb{P}_{\mathbb{C}}^{2}$ without fixed points nor invariant lines, it is possible to show that $\Omega(G)$ is the maximal open set where the action is properly discontinuous (see [Barrera Vargas et al. 2011]).

2.3. Classification of automorphisms of $\mathbb{P}_{\mathbb{C}}^{2}$. The nontrivial elements of $\operatorname{PGL}(3, \mathbb{C})$ can be classified as elliptic, parabolic or loxodromic (see [Navarrete 2008]). 
The elliptic elements in $\operatorname{PGL}(3, \mathbb{C})$ are those elements $g$ that have a lift to $\mathrm{GL}(3, \mathbb{C})$ whose Jordan canonical form is

$$
\left(\begin{array}{ccc}
e^{2 \pi i \theta_{1}} & 0 & 0 \\
0 & e^{2 \pi i \theta_{2}} & 0 \\
0 & 0 & e^{2 \pi i \theta_{3}}
\end{array}\right), \quad \text { where } \theta_{1}, \theta_{2}, \theta_{3} \in \mathbb{R}
$$

The limit set $\Lambda(g)$ for an elliptic element $g$ is $\varnothing$ or all of $\mathbb{P}_{\mathbb{C}}^{2}$ according to whether the order of $g$ is finite or infinite. Those subgroups of PGL $(3, \mathbb{C})$ containing an elliptic element of infinite order cannot be discrete.

The parabolic elements in PGL $(3, \mathbb{C})$ are those elements $g$ such that the limit set $\Lambda(g)$ is equal to a single complex line. If $g$ is parabolic then it has a lift to $\mathrm{GL}(3, \mathbb{C})$ whose Jordan canonical form is one of the following matrices:

$$
\left(\begin{array}{lll}
1 & 1 & 0 \\
0 & 1 & 0 \\
0 & 0 & 1
\end{array}\right), \quad\left(\begin{array}{lll}
1 & 1 & 0 \\
0 & 1 & 1 \\
0 & 0 & 1
\end{array}\right), \quad\left(\begin{array}{ccc}
e^{2 \pi i \theta} & 1 & 0 \\
0 & e^{2 \pi i \theta} & 0 \\
0 & 0 & e^{-4 \pi i \theta}
\end{array}\right), \quad \theta \in \mathbb{R} \backslash \mathbb{Z} .
$$

In the first case, $\Lambda(g)$ is the complex line consisting of all the fixed points of $g$. In the second case, $\Lambda(g)$ is the unique $g$-invariant complex line. In the last case, $\Lambda(g)$ is the complex line determined by the two fixed points of $g$.

There are four kinds of loxodromic elements in PGL $(3, \mathbb{C})$ :

- The complex homotheties are those elements $g \in \operatorname{PGL}(3, \mathbb{C})$ that have a lift to $\mathrm{GL}(3, \mathbb{C})$ whose Jordan canonical form is

$$
\left(\begin{array}{ccc}
\lambda & 0 & 0 \\
0 & \lambda & 0 \\
0 & 0 & \lambda^{-2}
\end{array}\right), \quad \lambda \in \mathbb{C}, \quad|\lambda| \neq 1,
$$

and its limit set $\Lambda(g)$ is the set of fixed points of $g$, consisting of one line $\ell$ and a point not lying in $\ell$. Moreover, in this case, $L_{0}(g) \cup L_{1}(g)=\Lambda(g)$ is not contained in one line.

- The screws are those elements $g \in \operatorname{PGL}(3, \mathbb{C})$ that have a lift to $\operatorname{GL}(3, \mathbb{C})$ whose Jordan canonical form is

$$
\left(\begin{array}{ccc}
\lambda & 0 & 0 \\
0 & \mu & 0 \\
0 & 0 & (\lambda \mu)^{-1}
\end{array}\right), \quad \lambda, \mu \in \mathbb{C}, \quad \lambda \neq \mu, \quad|\lambda|=|\mu| \neq 1,
$$

and its limit set $\Lambda(g)$ consists of the line $\ell$ on which $g$ acts as an elliptic transformation of $\operatorname{PSL}(2, \mathbb{C})$ and the fixed point of $g$ not lying in $\ell$. In this case, $L_{0}(g) \cup L_{1}(g)=\Lambda(g)$ is not contained in one line. 
- The loxoparabolic elements $g \in \operatorname{PGL}(3, \mathbb{C})$ have a lift to $\operatorname{GL}(3, \mathbb{C})$ whose Jordan canonical form is

$$
\left(\begin{array}{lll}
1 & 0 & 1 \\
0 & \lambda & 0 \\
0 & 0 & 1
\end{array}\right), \quad \lambda \in \mathbb{C}, \quad|\lambda| \neq 1,
$$

and the limit set $\Lambda(g)$ consists of two $g$-invariant complex lines. The element $g$ acts on one of these complex lines as a parabolic element of $\operatorname{PSL}(2, \mathbb{C})$ and on the other as a loxodromic element of $\operatorname{PSL}(2, \mathbb{C})$. In this case $L_{0}(g) \cup L_{1}(g)$ is contained in one line.

- The strongly loxodromic elements $g \in \operatorname{PGL}(3, \mathbb{C})$ have a lift to $\mathrm{GL}(3, \mathbb{C})$ whose Jordan canonical form is

$$
\left(\begin{array}{ccc}
\lambda_{1} & 0 & 0 \\
0 & \lambda_{2} & 0 \\
0 & 0 & \lambda_{3}
\end{array}\right), \quad \lambda_{1}, \lambda_{2}, \lambda_{3} \in \mathbb{C}, \quad\left|\lambda_{1}\right|<\left|\lambda_{2}\right|<\left|\lambda_{3}\right| .
$$

This kind of transformation has three fixed points: one of them is attracting, another is repelling and the last one is a saddle. The limit set $\Lambda(g)$ is equal to the union of the complex line determined by the attracting and saddle points and the complex line determined by the saddle and repelling points. In this case $L_{0}(g) \cup L_{1}(g)$ consists of three points in general position, so $L_{0}(g) \cup L_{1}(g)$ is not contained in one line.

Remark 2.4. If $g \in \operatorname{PGL}(3, \mathbb{C})$ satisfies that $L_{0}(g) \cup L_{1}(g)$ is contained in one line then $g$ is elliptic, parabolic or loxoparabolic.

2.4. Groups acting properly and discontinuously on $\mathbb{P}_{\mathbb{C}}^{2} \backslash \ell$. If $G$ is a subgroup of $\operatorname{PGL}(3, \mathbb{C})$ acting properly and discontinuously on $\mathbb{P}_{\mathbb{C}}^{2} \backslash \ell$, where $\ell \subset \mathbb{P}_{\mathbb{C}}^{2}$ is a line, then we can assume, from now on, that $\ell=\overleftrightarrow{e_{1}, e_{2}}$. So every element $g \in G$ can be induced by a matrix of the form

$$
\left(\begin{array}{lll}
a & b & v \\
c & d & w \\
0 & 0 & 1
\end{array}\right)
$$

When convenient, we shall write $a(g), b(g), c(g), \ldots$ instead of $a, b, c, \ldots$

We can regard $\mathbb{P}_{\mathbb{C}}^{2} \backslash \ell$ as $\mathbb{C}^{2}$, and (4) means that $g$ can be considered as the affine automorphism

$$
\mathbf{z} \mapsto A \mathbf{z}+\mathbf{v},
$$

where

$$
A=\left(\begin{array}{ll}
a & b \\
c & d
\end{array}\right), \quad \mathbf{v}=\left(\begin{array}{c}
v \\
w
\end{array}\right)
$$


The projection onto the linear part of the affine map above is denoted by

$$
\begin{gathered}
\phi: G \rightarrow \operatorname{PGL}(2, \mathbb{C}), \\
\phi(g)=A,
\end{gathered}
$$

and it is a group homomorphism.

On the other hand, the map

$$
\begin{gathered}
\psi: G \rightarrow \operatorname{PGL}(2, \mathbb{C}) \\
\psi(g)=\left(\begin{array}{ll}
a & v \\
0 & 1
\end{array}\right)
\end{gathered}
$$

is not necessarily a group homomorphism. However, it will be useful in Section 3 .

Given that $G$ acts properly discontinuously on $\mathbb{P}_{\mathbb{C}}^{2} \backslash \ell$, then for every $g \in G$, the cyclic group $\langle g\rangle$ acts properly and discontinuously on $\mathbb{P}_{\mathbb{C}}^{2} \backslash \ell$. So one has $L_{0}(g) \cup L_{1}(g) \subset \ell$. By Remark 2.4, $G$ contains only elliptic, parabolic or loxoparabolic elements.

In Section 3, we assume that $G$ contains a loxoparabolic element and we prove Theorem 1.1(i) together with some other results that will be useful for the proof of Theorem 1.2 in Section 4.

When $G$ does not contain any loxoparabolic elements, the group $\phi(G)$ contains only elliptic or parabolic elements. In the first part of Section 5, we consider the case when $G$ acts on $\ell$ without parabolic elements. In other words, $\phi(G)$ does not contain any parabolic element, and we prove Theorem 1.1(ii). Finally, in the last part of the same section, we consider the case when $\phi(G)$ contains a parabolic element, and we finish the proof of Theorem 1.1.

\section{3. $G$ contains a loxoparabolic element}

Lemma 3.1. Let $\ell$ be a line in $\mathbb{P}_{\mathbb{C}}^{2}$. If $G$ is a discrete subgroup of $\operatorname{PGL}(3, \mathbb{C})$ acting properly and discontinuously on $\mathbb{P}_{\mathbb{C}}^{2} \backslash \ell$ and $G$ contains a loxoparabolic element, then there exists a conjugate of $G$ such that every element in this conjugate group has a representative in $\mathrm{GL}(3, \mathbb{C})$ of the form

$$
\left(\begin{array}{lll}
a & 0 & v \\
0 & d & 0 \\
0 & 0 & 1
\end{array}\right),
$$

where a is a root of unity of order 1,2, 3, 4 or 6. Moreover, this conjugate group acts properly and discontinuously on $\mathbb{P}_{\mathbb{C}}^{2} \backslash \overleftrightarrow{e_{1}, e_{2}}$ 
Proof. Every element $g \in G$ has a representative matrix of the form (4) and we can assume that the matrix that induces a loxoparabolic element $h_{0}$ in $G$ has the form

$$
\left(\begin{array}{lll}
1 & 0 & 1 \\
0 & \lambda & 0 \\
0 & 0 & 1
\end{array}\right), \quad 0<|\lambda|<1
$$

The commutator $g_{n}=\left[h_{0}^{n}, g\right]$ and its inverse are induced by matrices of determinant one and their traces are equal to

$$
\tau_{n}=\frac{-3 a d+b c\left(1+\lambda^{-n}+\lambda^{n}\right)}{(b c-a d)} .
$$

If $b c \neq 0$ then, by Theorem 6.3 in [Navarrete 2008], $g_{n} \in G$ is a strongly loxodromic element for all sufficiently large $n$. Hence, for every $g \in G, b(g)=0$ or $c(g)=0$. It is not hard to check that $b(g)=0$ for every $g \in G$ or $c(g)=0$ for every $g \in G$. Therefore we can assume, conjugating if necessary, that $c(g)=0$ for every $g \in G$.

If $g \in G$ satisfies that $a(g)=1$ then, by Lemma 3.3 in [Fillmore and Scheuneman 1973], $g$ commutes with $h_{0}$. It follows that for every $h$ in the normal subgroup $H=\{g \in G: a(g)=1\}$, one has $b(h)=w(h)=0$.

Let $g$ be an arbitrary element in $G$. Then

$$
g h_{0} g^{-1}=\left(\begin{array}{ccc}
1 & \frac{b(-1+\lambda)}{d \lambda} & a-\frac{d+b w(-1+\lambda)}{d} \\
0 & d & w-w \lambda \\
0 & 0 & 1
\end{array}\right) \in H .
$$

It follows that $b(-1+\lambda) /(d \lambda)=0=w-w \lambda$. Hence $b=0=w$.

The line $\overleftrightarrow{e_{1}, e_{3}}$ is $G$-invariant because $b(g)=c(g)=w(g)=0$. Moreover, $G$ acts on it as a classic elementary group with limit point $e_{1}$. In fact, the action of $G$ on this line is the action on $\mathbb{P}_{\mathbb{C}}^{1}$ of the group

$$
\psi(G)=\left\{\left(\begin{array}{cc}
a(g) & v(g) \\
0 & 1
\end{array}\right): g \in G\right\},
$$

where $\psi$ is defined as in (7). It follows, by well-known facts on Euclidean groups (see [Maskit 1988]), that $a(g)$ is a root of unity of order 1, 2, 3, 4 or 6 .

Lemma 3.2. If $G \leq \mathrm{PGL}(3, \mathbb{C})$ acts properly and discontinuously on the complement of the line $\ell \subset \mathbb{P}_{\mathbb{C}}^{2}$ and $G$ contains a loxoparabolic element then $G$ contains a normal abelian subgroup $H$ isomorphic to $\mathbb{Z} \oplus \mathbb{Z}_{n_{0}}$ or to $\mathbb{Z} \oplus \mathbb{Z} \oplus \mathbb{Z}_{n_{0}}$ for some $n_{0} \in \mathbb{N}$. Moreover, the index of $H$ in $G$ is equal to $1,2,3,4$ or 6 .

Proof. We can assume that every element of $G$ is induced by a matrix of the form (8). In this case, the map $\psi: G \rightarrow \operatorname{PGL}(2, \mathbb{C})$, defined as in (7), is a homomorphism and 
its image is a Euclidean group of $\operatorname{PGL}(2, \mathbb{C})$. The kernel of this homomorphism consists of all those transformations in $G$ induced by a matrix of the form

$$
\left(\begin{array}{lll}
1 & 0 & 0 \\
0 & d & 0 \\
0 & 0 & 1
\end{array}\right)
$$

but these transformations are necessarily elliptic or the identity. Hence Ker $\psi$ is a finite group (because it is discrete and every element has finite order). Moreover, it is a cyclic group of some order $n_{0}$. Let us denote by

$$
\left(\begin{array}{ccc}
1 & 0 & 0 \\
0 & d_{0} & 0 \\
0 & 0 & 1
\end{array}\right)
$$

the generator of $\operatorname{Ker} \psi$.

Let $H$ be the normal abelian subgroup of $G$ consisting of those elements $g \in G$ induced by a matrix of the form

$$
\left(\begin{array}{lll}
1 & 0 & v \\
0 & d & 0 \\
0 & 0 & 1
\end{array}\right)
$$

We notice that $H / \operatorname{Ker} \psi$ is a free abelian subgroup of the Euclidean group $G / \operatorname{Ker} \psi$, consisting of all parabolic elements. Furthermore, $H / \operatorname{Ker} \psi$ has rank equal to one or two.

Since $H / \operatorname{Ker} \psi$ has index equal to $1,2,3,4$ or 6 in $G / \operatorname{Ker} \psi$, it follows that $H$ has index equal to $1,2,3,4$ or 6 in $G$.

Lemma 3.3. Let $G \leq \operatorname{PGL}(3, \mathbb{C})$ be a group that acts properly and discontinuously on $\mathbb{P}_{\mathbb{C}}^{2} \backslash \ell$. If $G$ contains a loxoparabolic element and the abelian normal subgroup $H$ has rank equal to one (where $H$ is as in Lemma 3.2), then $L_{0}(G)=L_{1}(G)$ consists of two points in $\ell$ and $L_{2}(G)$ is equal to the union of $\ell$ and one other line. In particular, the Kulkarni limit set of $G$ is equal to the union of two lines.

Proof. Let $\psi$ be as in (7) and $H$ be defined as the subgroup of $G$ induced by matrices of the form (9). There are two possible cases:

- If $H / \operatorname{Ker} \psi=G / \operatorname{Ker} \psi$, then we can assume that $G$ is generated by the two elements induced by the matrices

$$
\left(\begin{array}{lll}
1 & 0 & 1 \\
0 & \lambda & 0 \\
0 & 0 & 1
\end{array}\right), \quad\left(\begin{array}{ccc}
1 & 0 & 0 \\
0 & d_{0} & 0 \\
0 & 0 & 1
\end{array}\right),
$$

where $0<|\lambda|<1$ and $d_{0}$ is an $n_{0}$-th root of unity. 
- If $H / \operatorname{Ker} \psi \subsetneq G / \operatorname{Ker} \psi$, then we can assume that $G$ is generated by three elements induced by the matrices

$$
\left(\begin{array}{lll}
1 & 0 & 1 \\
0 & \lambda & 0 \\
0 & 0 & 1
\end{array}\right), \quad\left(\begin{array}{ccc}
1 & 0 & 0 \\
0 & d_{0} & 0 \\
0 & 0 & 1
\end{array}\right), \quad\left(\begin{array}{rcc}
-1 & 0 & 0 \\
0 & d_{0}^{m} & 0 \\
0 & 0 & 1
\end{array}\right),
$$

where $0<|\lambda|<1, d_{0}$ is an $n_{0}$-th root of unity and $m$ is an integer. (If the parabolic subgroup of a Euclidean group $E$ has rank one and it is not equal $E$, then $E$ is the dihedral infinite group $\{z \mapsto \pm z+n, n \in \mathbb{Z}\}$.)

In any case, the subgroup $N$ of $G$ generated by

$$
\left(\begin{array}{lll}
1 & 0 & 1 \\
0 & \lambda & 0 \\
0 & 0 & 1
\end{array}\right), \quad 0<|\lambda|<1,
$$

is normal and it has finite index in $G$. Moreover, $L_{0}(N)=L_{0}(G)=\left\{e_{1}, e_{2}\right\}$ and $L_{1}(G)=\bigcup_{i=1}^{m} g_{i}\left(L_{1}(N)\right)$, where $\left\{g_{1} N, \ldots, g_{m} N\right\}$ are all the cosets of $N$ in $G$, but $L_{1}(N)=\left\{e_{1}, e_{2}\right\}$ and each $g_{i}, i=1, \ldots, m$ fixes $e_{1}$ and $e_{2}$. Hence, $L_{1}(G)=\left\{e_{1}, e_{2}\right\}$.

Since $L_{0}(G)=L_{0}(N)=\left\{e_{1}, e_{2}\right\}=L_{1}(N)=L_{1}(G)$, it follows that

$$
L_{2}(G)=\bigcup_{i=1}^{m} g_{i}\left(L_{2}(N)\right)=\bigcup_{i=1}^{m} g_{i}\left(\overleftrightarrow{e_{1}, e_{2}} \cup \overleftrightarrow{e_{1}, e_{3}}\right)
$$

We conclude that $L_{2}(G)=\overleftrightarrow{e_{1}, e_{2}} \cup \overleftrightarrow{e_{1}, e_{3}}$ because $\overleftrightarrow{e_{1}, e_{2}} \cup \overleftrightarrow{e_{1}, e_{3}}$ is $G$-invariant

The proof of Theorem 1.1(i) follows immediately from Lemmas 3.2 and 3.3. Now we prove the converse.

Lemma 3.4. Let $G \leq \operatorname{PGL}(3, \mathbb{C})$ be a group that acts properly and discontinuously on $\mathbb{P}_{\mathbb{C}}^{2} \backslash \ell$. If $G$ contains a loxoparabolic element and the abelian normal subgroup $H$ has rank equal to two (where $H$ is as in Lemma 3.2), then $L_{0}(G) \cup L_{1}(G)=\ell$ and $L_{2}(G) \subset \ell$. In particular, the Kulkarni limit set of $G$ is equal to $\ell$.

Proof. We can assume that the two transformations induced by

$\gamma_{1}=\left(\begin{array}{lll}1 & 0 & 1 \\ 0 & \lambda & 0 \\ 0 & 0 & 1\end{array}\right), \quad 0<|\lambda|<1, \quad$ and $\quad \gamma_{2}=\left(\begin{array}{lll}1 & 0 & v \\ 0 & \delta & 0 \\ 0 & 0 & 1\end{array}\right), \quad v \in \mathbb{C} \backslash \mathbb{R}, \delta \in \mathbb{C}^{*}$,

generate a subgroup $N$ of finite index in the abelian group $H$. Hence, $L_{0}(N)=$ $L_{0}(H)=L_{0}(G)$ and $L_{1}(G)=\bigcup_{i=1}^{m} g_{i} L_{1}(N)$, where $\left\{g_{1} N, \ldots, g_{m} N\right\}$ are all distinct left cosets of $N$ in $G$. It follows that $L_{0}(G) \cup L_{1}(G)=\ell$ whenever $L_{0}(N) \cup L_{1}(N)=\ell$ because $\ell$ is $G$-invariant.

Now we consider all possible cases: 
If $|\delta|=1$, let us say $\delta=e^{2 i \pi \theta}, \theta \in \mathbb{R}$, then $L_{0}\left(\gamma_{2}\right)=\ell$ whenever $\theta \in \mathbb{Q}$ or $L_{1}\left(\gamma_{2}\right)=\ell$ whenever $\theta \notin \mathbb{Q}$. In any case, $L_{0}(N) \cup L_{1}(N)=\ell$.

If $|\delta| \neq 1$ then there are two cases depending on whether $\log |\lambda|$ and $\log |\delta|$ are rationally independent or not. In the first case, the action of $N$ on $\ell$ is not discrete. Hence $L_{0}(N) \cup L_{1}(N)=\ell$. In the second case, there exists an element $\gamma \in N$ such that $L_{0}(\gamma) \cup L_{1}(\gamma)=\ell$. Thus $L_{0}(N) \cup L_{1}(N)=\ell$.

Since $G$ acts properly and discontinuously on $\mathbb{P}_{\mathbb{C}}^{2} \backslash \ell$ and $L_{0}(G) \cup L_{1}(G)=\ell$, it follows (by Lemma 2.2) that $L_{2}(G) \subset \ell$.

We notice that the proof of Theorem 1.2(i) follows from Lemmas 3.3 and 3.4.

Examples. In these examples, $\lambda$ is a fixed complex number such that $0<|\lambda|<1$.

(i) The abelian group $G_{1}$, generated by the projective transformations

$$
\left(\begin{array}{lll}
1 & 0 & 1 \\
0 & \lambda & 0 \\
0 & 0 & 1
\end{array}\right), \quad\left(\begin{array}{lll}
1 & 0 & i \\
0 & 1 & 0 \\
0 & 0 & 1
\end{array}\right)
$$

acts properly and discontinuously on $\mathbb{C}^{2}=\mathbb{P}_{\mathbb{C}}^{2} \backslash \overleftrightarrow{e_{1}, e_{2}}$, and, by Lemma 3.4, $\Lambda\left(G_{1}\right)=\overleftrightarrow{e_{1}, e_{2}}$.

(ii) Let $\theta$ be a fixed real number. The abelian group $G_{2}$, generated by the projective transformations

$$
\left(\begin{array}{lll}
1 & 0 & 1 \\
0 & \lambda & 0 \\
0 & 0 & 1
\end{array}\right), \quad\left(\begin{array}{ccc}
1 & 0 & i \\
0 & e^{2 \pi i \theta} & 0 \\
0 & 0 & 1
\end{array}\right)
$$

acts properly and discontinuously on $\mathbb{C}^{2}=\mathbb{P}_{\mathbb{C}}^{2} \backslash \overleftrightarrow{e_{1}, e_{2}}$ and, by Lemma 3.4, $\Lambda\left(G_{2}\right)=\overleftrightarrow{e_{1}, e_{2}}$.

(iii) Let $\theta$ be a fixed real number and $n_{0} \in \mathbb{N}$ a fixed natural number. Denote by $G_{3}$ the abelian group $G_{3}$ generated by the projective transformations

$$
\left(\begin{array}{lll}
1 & 0 & 1 \\
0 & \lambda & 0 \\
0 & 0 & 1
\end{array}\right), \quad\left(\begin{array}{ccc}
1 & 0 & i \\
0 & e^{2 \pi i \theta} & 0 \\
0 & 0 & 1
\end{array}\right), \quad\left(\begin{array}{ccc}
1 & 0 & 0 \\
0 & e^{2 \pi i / n_{0}} & 0 \\
0 & 0 & 1
\end{array}\right) .
$$

Since $G_{2}$ is a finite-index subgroup of $G_{3}$, it follows that $G_{3}$ acts properly and discontinuously on $\mathbb{C}^{2}=\mathbb{P}_{\mathbb{C}}^{2} \backslash \overleftrightarrow{e_{1}, e_{2}}$. By Lemma $3.4, \Lambda\left(G_{3}\right)=\overleftrightarrow{e_{1}, e_{2}}$.

(iv) In an analogous way, the group generated by $G_{3}$ and the projective transformation

$$
\left(\begin{array}{lll}
i & 0 & 0 \\
0 & 1 & 0 \\
0 & 0 & 1
\end{array}\right)
$$

is a group whose Kulkarni limit set is equal to the line $\overleftrightarrow{e_{1}, e_{2}}$. 
(v) If $\theta \in \mathbb{R}$ and $n_{0} \in \mathbb{N}$ are fixed numbers, the reader can check that the group generated by the projective transformations

$$
\left(\begin{array}{ccc}
e^{\pi i / 3} & 0 & 0 \\
0 & 1 & 0 \\
0 & 0 & 1
\end{array}\right), \quad\left(\begin{array}{lll}
1 & 0 & 1 \\
0 & \lambda & 0 \\
0 & 0 & 1
\end{array}\right), \quad\left(\begin{array}{ccc}
1 & 0 & e^{\pi i / 3} \\
0 & e^{2 \pi i \theta} & 0 \\
0 & 0 & 1
\end{array}\right), \quad\left(\begin{array}{ccc}
1 & 0 & 0 \\
0 & e^{2 \pi i / n_{0}} & 0 \\
0 & 0 & 1
\end{array}\right)
$$

is a group whose Kulkarni limit set is equal to the line $\overleftrightarrow{e_{1}, e_{2}}$.

\section{Proof of Theorem 1.2}

The main purpose of this section is to prove Theorem 1.2. First, we prove some technical results in order to achieve our goal. In what follows we assume that $G$ does not contain any loxoparabolic elements.

Lemma 4.1. Let $G$ be a subgroup of $\operatorname{PGL}(3, \mathbb{C})$ acting properly and discontinuously on $\mathbb{P}_{\mathbb{C}}^{2} \backslash \ell$. If $G$ does not contain any loxoparabolic elements and $L_{0}(G) \cup L_{1}(G) \subsetneq \ell$, then $L_{2}(G)=\ell$.

Proof. Since $L_{0}(G) \cup L_{1}(G) \subsetneq \ell$, it follows that the group $\phi(G)$, where $\phi$ is defined as in (6), is a classical Kleinian group containing only parabolic or elliptic elements. Hence it acts as an elementary group on $\ell$. It follows that this group has a fixed point in $\ell$, so we can assume this fixed point is $e_{1}$, and it implies that every element in $G$ can be represented by a matrix of the form

$$
\left(\begin{array}{llc}
a & b & v \\
0 & d & w \\
0 & 0 & 1
\end{array}\right)
$$

The kernel of the homomorphism $\phi: G \rightarrow \operatorname{PGL}(2, \mathbb{C})$ consists of all the transformations in $G$ induced by matrices of the form

$$
\left(\begin{array}{lll}
a & 0 & v \\
0 & a & w \\
0 & 0 & 1
\end{array}\right)
$$

Since $L_{0}(G) \cup L_{1}(G) \subsetneq \ell$, every infinite order element in $G$ has canonical form

$$
\left(\begin{array}{lll}
1 & 1 & 0 \\
0 & 1 & 1 \\
0 & 0 & 1
\end{array}\right)
$$

It follows that every element in $\operatorname{Ker} \phi$ is elliptic, and so $\operatorname{Ker} \phi$ is finite. Moreover, it is cyclic because it is isomorphic to a finite subgroup of $\mathbb{S}^{1}$. This isomorphism is 
given by

$$
\left(\begin{array}{ccc}
a & 0 & v \\
0 & a & w \\
0 & 0 & 1
\end{array}\right) \mapsto a .
$$

Lemma 4.2. Let $G$ be a subgroup of $\operatorname{PGL}(3, \mathbb{C})$ acting properly and discontinuously on $\mathbb{P}_{\mathbb{C}}^{2} \backslash \ell$. If $G$ does not contain any loxoparabolic elements and $L_{0}(G) \cup L_{1}(G) \subsetneq \ell$, then the matrix

$$
Q=\left(\begin{array}{lll}
0 & 0 & 1 \\
0 & 0 & 0 \\
0 & 0 & 0
\end{array}\right)
$$

induces the only pseudo-projective limit of the group $G$, also denoted by $Q$. In consequence, by Lemma 2.2, $L_{2}(G) \subset \ell$.

Proof. Let $g_{n}$ be a sequence of distinct elements in $G$ such that $g_{n} \rightarrow R$ when $n \rightarrow \infty$ ( where $R$ is a pseudo-projective transformation)

Case 1. The sequence contains a subsequence which consists only of parabolic elements.

In this case, we can assume that the elements are induced by matrices of the form

$$
\left(\begin{array}{ccc}
1 & b_{n} & v_{n} \\
0 & 1 & w_{n} \\
0 & 0 & 1
\end{array}\right),
$$

but the set of all these transformations lying in $G$ form a subgroup of $G$ which is abelian because the commutator of two such elements is equal to the identity or to a parabolic element having a line of fixed points (and the group $G$ does not contain this kind of parabolic elements). Moreover, the restriction of the homomorphism $\phi$ to this abelian subgroup is an isomorphism onto the "parabolic subgroup" of the Euclidean group $\{A(g): g \in G\}$. Hence, this free abelian subgroup has rank at most two. If the group has rank one, then we can assume (conjugating by an upper triangular matrix) that this group is generated by an element induced by a matrix of the form

$$
\left(\begin{array}{lll}
1 & 1 & 0 \\
0 & 1 & 1 \\
0 & 0 & 1
\end{array}\right),
$$

and it is not hard to check that the pseudo-projective limit $R$ is induced by the matrix $Q$. 
If the rank is equal to two, then we can assume (conjugating by an upper triangular matrix) that the parabolic group is generated by

$$
A=\left(\begin{array}{lll}
1 & 1 & 0 \\
0 & 1 & 1 \\
0 & 0 & 1
\end{array}\right), \quad B=\left(\begin{array}{lll}
1 & \beta & v \\
0 & 1 & \beta \\
0 & 0 & 1
\end{array}\right), \quad \beta \in \mathbb{C} \backslash \mathbb{R} .
$$

It is not hard to check, by means of an analysis of the general element $A^{m} B^{n}$ that any pseudo-projective limit must be induced by the matrix $Q$.

Case 2. The sequence $\left(g_{n}\right)$ consists (except for finitely many values of $n$ ) of elliptic elements.

Since $G / \operatorname{Ker} \phi$ is a Euclidean group and $\operatorname{Ker} \phi$ is finite, there is an $n_{0} \in \mathbb{N}$ such that for every elliptic element in $G, g^{n_{0}}=\mathrm{Id}$. Therefore, there exists a subsequence of $\left(g_{n}\right)$, still denoted $\left(g_{n}\right)$, and a diagonal matrix $h$ such that $\left(h^{-1} g_{n}\right)$ is a sequence of distinct parabolic elements (because these transformations are represented by upper triangular matrices with ones in the diagonal entries). By Case 1 above, it follows that the pseudo-projective limit of $h^{-1} g_{n}$ is induced by the matrix $Q$. Therefore, the pseudo-projective limit of the sequence $g_{n}$ is induced by the same matrix.

Finally, if $\ell_{0}$ is a line not intersecting $L_{0}(G) \cup L_{1}(G)$ and $g \in G$ is a parabolic element, then the family of compact sets $\left\{g^{n}\left(\ell_{0}\right)\right\}$ accumulates in $\ell$. Therefore, $\ell \subset L_{2}(G)$.

Proof of Theorem 1.2. The proof of (i) follows from Lemmas 3.3 and 3.4.

Now, for (ii), the group $G$ contains a parabolic element of infinite order. Since $G$ acts properly and discontinuously on $\mathbb{P}_{\mathbb{C}}^{2} \backslash \ell$, it follows that $L_{0}(G) \cup L_{1}(G) \subset \ell$.

Then, we consider two cases according to whether $L_{0}(G) \cup L_{1}(G)=\ell$ or $L_{0}(G) \cup L_{1}(G) \subsetneq \ell$.

If $L_{0}(G) \cup L_{1}(G)=\ell$, then, by Lemma 2.2, $L_{2}(G) \subset \ell$. Therefore, $\ell=\Lambda(G)$.

If $L_{0}(G) \cup L_{1}(G) \subsetneq \ell$, then by Lemma 4.1, $L_{2}(G)=\ell$.

The next corollary follows from Lemma 4.2.

Corollary 4.3. If $G \subset \operatorname{PGL}(3, \mathbb{C})$ acts properly and discontinuously on $\mathbb{P}_{\mathbb{C}}^{2} \backslash \ell$ and $G$ does not contain any loxoparabolic elements, then one and only one of the following statements is verified:

- $L_{0}(G) \cup L_{1}(G)=\ell$ and $L_{2}(G) \subset \ell$, or

- $L_{0}(G)=L_{1}(G)$ is a point in $\ell$ and $L_{2}(G)=\ell$.

Proposition 4.4. If $G \subset \operatorname{PGL}(3, \mathbb{C})$ is a discrete subgroup such that $\Lambda(G)$ is equal to a line $\ell$ and $G$ does not contain loxoparabolic elements, then for any finite extension $G_{1}$ of $G$ (i.e., $G$ is a finite-index normal subgroup of $G_{1} \leq \operatorname{PGL}(3, \mathbb{C})$ ), $\Lambda\left(G_{1}\right)=\Lambda(G)=\ell$. 
Proof. (a) Since $G$ is a finite-index normal subgroup of $G_{1}$, we have $L_{0}(G)=$ $L_{0}\left(G_{1}\right)$ and $L_{1}\left(G_{1}\right)=g_{1}\left(L_{1}(G)\right) \cup \cdots \cup g_{k}\left(L_{1}(G)\right)$, where $\left[g_{1}\right], \ldots,\left[g_{k}\right]$ are all the distinct cosets in $G_{1} / G$.

(b) If $L_{0}\left(G_{1}\right)=L_{0}(G)$ and $L_{1}\left(G_{1}\right)=L_{1}(G)$ then

$$
L_{2}\left(G_{1}\right)=g_{1}\left(L_{2}(G)\right) \cup \cdots \cup g_{k}\left(L_{2}(G)\right),
$$

where $\left[g_{1}\right], \ldots,\left[g_{k}\right]$ are all the distinct cosets in $G_{1} / G$.

(c) If $L_{0}(G) \cup L_{1}(G)=\ell$ then we consider two cases.

Case 1. The set $L_{0}(G)$ consists of a single point, called $p$. In this case, $L_{0}\left(G_{1}\right) \cup$ $L_{1}\left(G_{1}\right)$ is a $G_{1}$-invariant set and it is a finite union of lines passing through $p$ (the line $\ell$ is one of such lines). Let $g \in G_{1}$ be a parabolic element with $p$ as its only fixed point. Then there exists $n_{0} \in \mathbb{N}$ such that every line in $L_{0}\left(G_{1}\right) \cup L_{1}\left(G_{1}\right)$ is $g^{n_{0}}$-invariant. Since $g^{n_{0}}$ is parabolic with a single fixed point, it has a single invariant line. Hence $L_{0}\left(G_{1}\right) \cup L_{1}\left(G_{1}\right)=\ell$.

Case 2. The set $L_{0}(G)$ contains more than one point. In this case, the set $L_{0}\left(G_{1}\right)=$ $L_{0}(G)$ determines the line $\ell$ and is $G_{1}$-invariant. Then $\ell$ is $G_{1}$-invariant and

$$
L_{0}\left(G_{1}\right) \cup L_{1}\left(G_{1}\right)=g_{1}\left(L_{0}(G) \cup L_{1}(G)\right) \cup \cdots \cup g_{k}\left(L_{0}(G) \cup L_{1}(G)\right)=\ell .
$$

In any case, it is not hard to check that $G_{1}$ acts properly and discontinuously on $\mathbb{P}_{\mathbb{C}}^{2} \backslash \ell$, and by Lemma 2.2 we obtain that $L_{2}\left(G_{1}\right) \subset \ell$.

(d) If $L_{0}(G) \cup L_{1}(G) \subsetneq \ell$, then by Corollary 4.3, $L_{0}(G)=L_{1}(G)$ is equal to one point. Thus we can assume that $L_{2}(G)=\ell$ and $L_{0}(G)=L_{1}(G)=\left\{e_{1}\right\}$. In this case, $L_{0}\left(G_{1}\right)=L_{0}(G)=\left\{e_{1}\right\}$ and it follows that every element in $G_{1}$ fixes $e_{1}$. Hence (by item (a)), $L_{1}\left(G_{1}\right)=L_{1}(G)=\left\{e_{1}\right\}$. It follows (by item (b)) that $L_{2}\left(G_{1}\right)=g_{1}(\ell) \cup \cdots \cup g_{k}(\ell)$, where $\left[g_{1}\right], \ldots,\left[g_{k}\right]$ are all distinct cosets in $G_{1} / G$. Therefore, $\Lambda\left(G_{1}\right)=\ell_{1} \cup \cdots \cup \ell_{k}$, but there exists a parabolic element $g_{0} \in G \subset G_{1}$ with Jordan canonical form equal to

$$
\left(\begin{array}{lll}
1 & 1 & 0 \\
0 & 1 & 1 \\
0 & 0 & 1
\end{array}\right) .
$$

Thus, every line $\ell_{j}, j=1, \ldots, k$, is invariant under some fixed power of $g_{0}$, but every power of $g_{0}$ has a single invariant line, and this line is equal to $\ell$. We conclude that $\Lambda\left(G_{1}\right)=\ell$.

\section{Proof of Theorem 1.1}

We recall that the proof of Theorem 1.1(i) follows from Lemmas 3.2 and 3.3. 
Proof of Theorem 1.1(ii). Since $G$ does not contain any element which acts as a parabolic element on $\ell$, it follows that $\phi(G) \backslash\{\mathrm{Id}\}$ induces only elliptic elements, where $\phi$ is defined as in (6). We notice that the determinant of the matrix

$$
A=\left(\begin{array}{ll}
a & b \\
c & d
\end{array}\right)
$$

has modulus equal to one. Hence, every element in $\phi(G)$ can be written as a matrix of the form

$$
\lambda\left(\begin{array}{ll}
a & b \\
c & d
\end{array}\right), \quad \lambda \in \mathbb{S}^{1} \quad \text { and } \quad a d-b c=1 .
$$

Since $\phi(G)$ induces a purely elliptic group acting on $\hat{\mathbb{C}}$, there is a global fixed point for the action of $\phi(G)$ on $\mathbb{T}_{\mathbb{R}}^{3}$. We can assume that this global fixed point is $(0,0,1)$ (in the upper half-space model). Then every element in $\phi(G)$ can be written as a matrix of the form

$$
\lambda\left(\begin{array}{rr}
a & b \\
-\bar{b} & \bar{a}
\end{array}\right), \quad \lambda \in \mathbb{S}^{1} \quad \text { and } \quad|a|^{2}+|b|^{2}=1 .
$$

Therefore, every element $g \in G$ can be written in the form

$$
g=\left(\begin{array}{ll}
A & \mathbf{v} \\
0 & 1
\end{array}\right), \quad \text { where } A \in U(2) \quad \text { and } \quad \mathbf{v}, 0^{\mathrm{T}} \in \mathbb{C}^{2} .
$$

Hence, $G$ is a discrete subgroup of isometries of $\mathbb{R}^{4}$. By Theorem 5.4.5 in [Ratcliffe 1994], $G$ contains a normal finite-index free abelian subgroup of rank less than or equal to four.

Remark 5.1. If $\phi(G) \backslash\{\mathrm{Id}\}$ induces only elliptic elements, then $G$ is conjugate in $\operatorname{PGL}(3, \mathbb{C})$ to some group such that every element in a finite-index subgroup is induced by an upper triangular matrix.

Examples. (i) Let $G$ be the group generated by the two matrices

$$
\left(\begin{array}{ccc}
1 & 0 & 1 \\
0 & e^{2 \pi i \theta_{1}} & 0 \\
0 & 0 & 1
\end{array}\right), \quad\left(\begin{array}{ccc}
1 & 0 & i \\
0 & e^{2 \pi i \theta_{2}} & 0 \\
0 & 0 & 1
\end{array}\right)
$$

where $\theta_{1}, \theta_{2}$ are fixed real numbers. It is not hard to check that $G$ acts properly and discontinuously on $\mathbb{P}_{\mathbb{C}}^{2} \backslash \overleftrightarrow{e_{1}, e_{2}}$. It follows from Theorem 1.2(ii) that $\Lambda(G)=\overleftrightarrow{e_{1}, e_{2}}$. In this case $\phi(G)$ does not contain any parabolic elements.

(ii) Let $G_{1}$ be the group generated by the matrices

$$
\left(\begin{array}{lll}
1 & 0 & 1 \\
0 & 1 & 0 \\
0 & 0 & 1
\end{array}\right), \quad\left(\begin{array}{lll}
1 & 0 & i \\
0 & 1 & 0 \\
0 & 0 & 1
\end{array}\right), \quad\left(\begin{array}{lll}
1 & 0 & 0 \\
0 & 1 & 1 \\
0 & 0 & 1
\end{array}\right), \quad\left(\begin{array}{lll}
1 & 0 & 0 \\
0 & 1 & i \\
0 & 0 & 1
\end{array}\right) .
$$


It is not hard to check that $G_{1}$ acts properly and discontinuously on $\mathbb{P}_{\mathbb{C}}^{2} \backslash \overleftrightarrow{e_{1}, e_{2}}$. It follows from Theorem 1.2(ii) that $\Lambda\left(G_{1}\right)=\overleftrightarrow{e_{1}, e_{2}}$. In this case $\phi\left(G_{1}\right)$ consists of the identity element.

(iii) If $k$ is a fixed natural number, then the group $G_{2}$ generated by the matrices

$$
\left(\begin{array}{lll}
1 & 0 & 0 \\
0 & 1 & k \\
0 & 0 & 1
\end{array}\right), \quad\left(\begin{array}{rrr}
1 & -1 & 0 \\
0 & 1 & i \\
0 & 0 & 1
\end{array}\right), \quad\left(\begin{array}{lll}
1 & 0 & 1 \\
0 & 1 & 0 \\
0 & 0 & 1
\end{array}\right), \quad\left(\begin{array}{lll}
1 & 0 & i \\
0 & 1 & 0 \\
0 & 0 & 1
\end{array}\right)
$$

acts properly and discontinuously on $\mathbb{P}_{\mathbb{C}}^{2} \backslash \overleftrightarrow{e_{1}, e_{2}}$ and the Kulkarni limit set $\Lambda\left(G_{2}\right)$ is equal to $\overleftrightarrow{e_{1}, e_{2}}$ by Theorem 1.2(ii). In this case, $\phi\left(G_{2}\right)$ is a cyclic group generated by a parabolic element. We notice that $G_{2}$ is a 2-step nilpotent group, so it is a uniform lattice of $H_{3} \times \mathbb{R}$, where $H_{3}$ is the 3-dimensional real Heisenberg group (see [Dekimpe 1996, Corollary 6.2.5]). If $\Gamma$ is a subgroup of $G_{2}$ such that $0<\operatorname{rank} \Gamma<4$ then $\Lambda(\Gamma)=\overleftrightarrow{e_{1}, e_{2}}$. However, the quotient of $\mathbb{P}_{\mathbb{C}}^{2} \backslash \overleftrightarrow{e_{1}, e_{2}}$ by $\Gamma$ is not compact.

In what follows we develop some tools that will be useful in the proof of Theorem 1.1(iii).

Proposition 5.2. If $G \subset \operatorname{PGL}(3, \mathbb{C})$ is a discrete subgroup such that $\Lambda(G)$ is equal to a line $\ell, G$ does not contain any loxoparabolic elements and $G$ contains an element which acts as a parabolic element on $\ell$, then $G$ is conjugate in $\operatorname{PGL}(3, \mathbb{C})$ to some group such that every element in it is induced by a matrix of the form

$$
\left(\begin{array}{llc}
a & b & v \\
0 & d & w \\
0 & 0 & 1
\end{array}\right), \quad|a|=|d|=1 .
$$

(If the group $G$ does contain any loxoparabolic elements, then the statement is still valid with the exception that the eigenvalues are not unitary complex numbers. See Lemma 3.1)

Proof. Since the set $\phi(G)$ contains a parabolic element, we can assume (conjugating, if needed, by an element that preserves the line $\ell$ ) that such parabolic element is induced by a matrix of the form

$$
A_{1}=\mu\left(\begin{array}{ll}
1 & 1 \\
0 & 1
\end{array}\right) \quad \text { for some } \mu \in \mathbb{C}
$$

If we assume there is a matrix

$$
A_{2}=\left(\begin{array}{ll}
a & b \\
c & d
\end{array}\right) \quad \text { such that } c \neq 0
$$


which induces an element in $\phi(G)$, then $\operatorname{Tr}^{2}\left(A_{1}^{n} A_{2} A_{1}^{-n} A_{2}^{-1}\right)=\left(2+(c n)^{2}\right)^{2}$. Thus, $A_{1}^{n} A_{2} A_{1}^{-n} A_{2}^{-1}$ is a loxodromic element for $n$ large enough, and it contradicts that $\phi(G)$ contains only elliptic or parabolic elements.

Lemma 5.3. Let $V$ be an abelian subgroup of $\mathbb{C}^{2}$ of rank $r=1,2,3$ or 4 and $u$ an $m$-th root of unity that generates a cyclic group of order $m$. Assume that $u v \in V$ whenever $v \in V$. Then $\varphi(m) \leq r$ (where $\varphi$ denotes the Euler's totient function). Hence, $m \leq 12$.

Proof. This proof is contained in the proof of Theorem 4.1 in [Fillmore and Scheuneman 1973], and we include it here for reader's convenience. Let $v_{1}, \ldots, v_{r}$ be a basis of $V$. Expressing $u v_{i}$ in terms of this basis and taking a determinant, we obtain a polynomial of degree $r$ with integer coefficients which is satisfied by $u$. Hence the field generated by $u$ over the rationals is of degree at most $r$. This field is generated by a primitive $m$-th root of unity, so it has degree $\varphi(m)$, where $\varphi$ is Euler's totient. Thus $\varphi(m) \leq r$. It follows that $m=1,2,3,4,5,6,8,10$ or 12 .

The hypothesis in the lemma above can be slightly modified to obtain the following:

Lemma 5.4. Let $V$ be an abelian subgroup of $\mathbb{C}^{2}$ of rank $r=1,2$ or 3 and $u \in \mathbb{S}^{1}$. Assume $u v \in V$ whenever $v \in V$. Then $u$ is a root of unity of order 1, 2, 3, 4 or 6 .

The proof is almost the same as the one given for Lemma 5.3. One just uses the fact that $u \in \mathbb{S}^{1}$ is a root of unity whenever there is a monic polynomial of degree $r \leq 3$ with integer coefficients which is satisfied by $u$.

Remark 5.5. Lemmas 5.3 and 5.4 can be applied to abelian subgroups of

$$
(\mathbb{C},+) \cong(\{0\} \times \mathbb{C},+) \cong(\mathbb{C} \times\{0\},+) .
$$

If we assume that every element in $G$ can be represented by an upper triangular matrix of the form (10) and $G$ contains an irrational ellipto-parabolic element then we can assume that it is induced by one of the following two upper triangular matrices:

$$
E_{1}=\left(\begin{array}{ccc}
1 & 0 & 1 \\
0 & d_{0} & 0 \\
0 & 0 & 1
\end{array}\right), E_{2}=\left(\begin{array}{ccc}
a_{0} & 0 & 0 \\
0 & 1 & 1 \\
0 & 0 & 1
\end{array}\right), \quad a_{0}=d_{0}=e^{2 \pi i \theta}, \theta \in \mathbb{R} \backslash \mathbb{Q} .
$$

Proposition 5.6. Let $G$ be a discrete subgroup of $\operatorname{PGL}(3, \mathbb{C})$ such that every element can be induced by a matrix of the form $(10)$ and $\Lambda(G)=\ell$. If $G$ contains the irrational ellipto-parabolic element induced by $E_{1}$, then the normal subgroup $N_{1}$ 
consisting of the elements of the form

$$
\left(\begin{array}{lll}
1 & b & v \\
0 & d & w \\
0 & 0 & 1
\end{array}\right)
$$

is a (finitely generated) finite-index abelian subgroup. In fact, $b=w=0$ for every element in $N_{1}$.

Proof. Every element in the subgroup $N_{1}$ commutes with $E_{1}$ (by Lemma 3.3 in [Fillmore and Scheuneman 1973]), and hence it has the form

$$
h=\left(\begin{array}{ccc}
1 & 0 & v(h) \\
0 & d(h) & 0 \\
0 & 0 & 1
\end{array}\right) .
$$

Therefore, $N_{1}$ is a finitely generated abelian subgroup. Moreover, the set

$$
V=\left\{v \in \mathbb{C} \mid \exists h \in N_{1} \text { with } v(h)=v\right\}
$$

is an abelian subgroup of $(\mathbb{C},+)$.

Now, let $g$ be any element in $G$. By considering the upper right entry of $g h g^{-1}$, it is possible to check that $a(g) v \in V$ whenever $v \in V$. It follows, by Lemma 5.4, that $a(g)$ is a root of unity of order $1,2,3,4$ or 6 . Therefore $N_{1}$ has finite index in $G$. Since every element in the discrete abelian group $N_{1}$ has the form (11), it is not hard to check that $N_{1}$ is finitely generated and its rank is less or equal to two.

Proposition 5.7. Let $G$ be a discrete subgroup of $\operatorname{PGL}(3, \mathbb{C})$ such that every element can be induced by a matrix of the form (10) and $\Lambda(G)=\ell$. If $G$ contains the irrational ellipto-parabolic element induced by $E_{2}$, then the normal subgroup $\mathrm{N}_{2}$ consisting of those elements of the form

$$
\left(\begin{array}{ccc}
a & b & v \\
0 & 1 & w \\
0 & 0 & 1
\end{array}\right)
$$

is a (finitely generated) finite-index abelian subgroup. In fact, $b=v=0$ for every element in $\mathrm{N}_{2}$.

Proof. Let us denote by $L$ the set consisting of those $(\beta, v) \in \mathbb{C}^{2}$ for which there exists an element in $G$ of the form

$$
\left(\begin{array}{lll}
1 & \beta & v \\
0 & 1 & 0 \\
0 & 0 & 1
\end{array}\right)
$$


It is not hard to check that $L$ is a discrete subgroup of $\left(\mathbb{C}^{2},+\right)$. If we assume that $b \neq 0$ for some

$$
f=\left(\begin{array}{ccc}
a & b & v \\
0 & 1 & w \\
0 & 0 & 1
\end{array}\right) \in N_{2}
$$

then $L \neq \mathbf{0}$ because the commutator of $E_{2}$ and $f$ is equal to

$$
\left[E_{2}, f\right]=\left(\begin{array}{ccc}
1 & \left(a_{0}-1\right) b & \left(a_{0}-1\right) v-\left(a_{0}-1\right) b w-a_{0} b \\
0 & 1 & 0 \\
0 & 0 & 1
\end{array}\right) .
$$

If $(\beta, v) \in L$ then $\left(a_{0} \beta, a_{0} v-a_{0} \beta\right) \in L$ (conjugate the element of the form (12) by $\left.E_{2}\right)$. In other words, the $\mathbb{C}$-linear map induced by

$$
\left(\begin{array}{rr}
a_{0} & 0 \\
-a_{0} & a_{0}
\end{array}\right)
$$

preserves $L$. If we assume $\operatorname{rank} L=4$, then $a_{0}$ is a root of a polynomial with integer coefficients of the form $p(x)=\left(x^{2}-2 x \cos (2 \pi \theta)+1\right)^{2}$ and it implies that $a_{0}$ is a root of unity, which is a contradiction. Therefore, $\operatorname{rank} L \leq 3$. Hence, applying Lemma 5.4 to the abelian group $L$ and to the unitary complex number $a_{0}$, we obtain that $a_{0}$ is a root of unity, a contradiction. It follows that $b=0$ for all $f \in N_{2}$.

Analogously, it can be proved that $v=0$ for all $f \in N_{2}$. Thus every $f \in N_{2}$ has the form

$$
\left(\begin{array}{ccc}
a & 0 & 0 \\
0 & 1 & w \\
0 & 0 & 1
\end{array}\right)
$$

and it follows that $N_{2}$ is an abelian group of rank less than or equal to two.

Let $W$ denote the subset of $\mathbb{C}$ consisting of those $\omega \in \mathbb{C}$ such that there exist $f \in N_{2}$ of the form

$$
f=\left(\begin{array}{ccc}
a & 0 & 0 \\
0 & 1 & \omega \\
0 & 0 & 1
\end{array}\right)
$$

We notice that $W$ is an abelian discrete subgroup of $\mathbb{C}$ and $W \neq 0$ because $E_{2} \in N_{2}$. If $g \in G$ is an arbitrary element, then it is not hard to check that $d(g) \omega \in W$ whenever $\omega \in W$. It follows, by Lemma 5.4, that $d(g)$ is a root of unity of order 1, 2, 3, 4 or 6 . Therefore $N_{2}$ has finite index in $G$.

Proposition 5.8. Let $G$ be a discrete subgroup of $\operatorname{PGL}(3, \mathbb{C})$ such that $\Lambda(G)=\ell$ and every element $g \in G$ is induced by a matrix of the form (10). Assume that for every $g \in G$, there exists $n \in \mathbb{N}$, depending on $g$, such that $a(g), d(g)$ are $n$-th roots 
of unity. If $N$ denotes the subset of $G$ consisting of those elements in $G$ induced by matrices of the form

$$
\left(\begin{array}{ccc}
1 & b & v \\
0 & 1 & w \\
0 & 0 & 1
\end{array}\right),
$$

then $N$ is a finite-index torsion free normal subgroup of $G$ (it is also finitely generated).

Proof. Let $B$ be the abelian subgroup of $\mathbb{C}$ that consists of all those $\beta \in \mathbb{C}$ for which there exists an element in $N$ of the form

$$
\left(\begin{array}{ccc}
1 & \beta & v \\
0 & 1 & w \\
0 & 0 & 1
\end{array}\right) .
$$

Analogously, let $W$ be the abelian subgroup of $\mathbb{C}$ consisting of those $\omega \in \mathbb{C}$ such that exists an element in $N$ of the form

$$
\left(\begin{array}{lll}
1 & b & v \\
0 & 1 & \omega \\
0 & 0 & 1
\end{array}\right) .
$$

Finally, let $V$ be the subset of $\mathbb{C}$ (it is not necessarily a subgroup) consisting of those $v \in \mathbb{C}$ such that there exists an element in $G$ of the form

$$
\left(\begin{array}{ccc}
1 & b & v \\
0 & 1 & w \\
0 & 0 & 1
\end{array}\right) .
$$

Since $G$ acts properly and discontinuously on $\mathbb{C}^{2}$, so does $N$. Moreover, the nilpotent group $N$ acts freely on $\mathbb{C}^{2}$, so $N$ is generated by at most four elements (see [Cartan and Eilenberg 1999] and [Fillmore and Scheuneman 1973]). It follows that rank $B \leq 4$ and rank $W \leq 4$. Moreover, when $V$ is an abelian group, rank $V \leq 4$.

We consider the following cases:

Case 1. $B \neq 0$ and $W \neq 0$.

In this case, by Lemma 5.3 applied to $W$ and to the root of unity $d(g)$, there exists $n_{1} \in \mathbb{N}$ (not depending on $g$ ) such that for every $g \in G, d(g)^{n_{1}}=1$.

Similarly, by Lemma 5.3 applied to $B$ and to the root of unity $a(g) / d(g)$, there exists $n_{2} \in \mathbb{N}$ (not depending on $g$ ) such that for every $g \in G,(a(g) / d(g))^{n_{2}}=1$.

Therefore, there exists $n_{0} \in \mathbb{N}$ such that $g^{n_{0}} \in N$ for every $g \in G$.

Case 2. $B \neq 0, W=0$ and $V \neq 0$.

In this case, by Lemma 5.3 applied to the abelian group $V$ and to the root of unity $a(g) / d(g)$, there exists $n_{1} \in \mathbb{N}$ such that $(a(g) / d(g))^{n_{1}}=1$ for every $g \in G$. 
Let us fix an element

$$
g_{0}=\left(\begin{array}{ccc}
a_{0} & b_{0} & v_{0} \\
0 & d_{0} & w_{0} \\
0 & 0 & 1
\end{array}\right) \in G,
$$

with $d_{0} \neq 1$ (if such an element does not exist then the proof ends). If $g \in G$ is an arbitrary element, then the commutator $\left[g_{0}, g\right]$ has the form

$$
\left(\begin{array}{ccc}
1 & \beta & v \\
0 & 1 & \left(d_{0}-1\right) w-(d-1) w_{0} \\
0 & 0 & 1
\end{array}\right) .
$$

Then $\left(d_{0}-1\right) w-(d-1) w_{0}=0$. Therefore, conjugating $G$ by the projective transformation induced by

$$
h=\left(\begin{array}{ccc}
1 & 0 & 0 \\
0 & d_{0}-1 & w_{0} \\
0 & 0 & 1
\end{array}\right),
$$

we can assume that $w=0$ for all $g \in G$.

Now, if $v \in V$ and $g$ is an arbitrary element in $G$ as above, then $a v \in V$ (just conjugate

$$
\left(\begin{array}{lll}
1 & \beta & v \\
0 & 1 & 0 \\
0 & 0 & 1
\end{array}\right)
$$

by $g$ ). It follows, by Lemma 5.3, that there exists $n_{2} \in \mathbb{N}$ such that $a^{n_{2}}=1$ for every $g \in G$. Therefore, there exists $n_{0} \in \mathbb{N}$ such that $g^{n_{0}} \in N$ for every $g \in G$.

Case 3. $B \neq 0, W=0$ and $V=0$.

This case cannot happen, otherwise the Kulkarni limit set of $N$ (and the Kulkarni limit set of $G$ ) would be equal to $\overleftrightarrow{e_{1}, e_{3}}$.

Case 4. $B=0, W \neq 0$ and $V \neq 0$.

In this case, $V$ is an abelian subgroup of $\mathbb{C}$. By Lemma 5.3, there exists $n_{1} \in \mathbb{N}$ such that $d^{n_{1}}=1$ for every $g \in G$. Let us fix an element

$$
g_{0}=\left(\begin{array}{ccc}
a_{0} & b_{0} & v_{0} \\
0 & d_{0} & w_{0} \\
0 & 0 & 1
\end{array}\right) \in G,
$$

with $a_{0} \neq d_{0}$ (if such element does not exist then the proof ends). 
If $g \in G$ is an arbitrary element, then the commutator $\left[g_{0}, g\right]$ has the form

$$
\left(\begin{array}{ccc}
1 & \frac{b\left(a_{0}-d_{0}\right)-b_{0}(a-d)}{d d_{0}} & v \\
0 & 1 & \omega \\
0 & 0 & 1
\end{array}\right) .
$$

Then $\left[g_{0}, g\right] \in N$, so $b\left(a_{0}-d_{0}\right)-b_{0}(a-d)=0$.

Hence, we can assume, conjugating $G$ by

$$
h=\left(\begin{array}{ccc}
a_{0}-d_{0} & b_{0} & 0 \\
0 & 1 & 0 \\
0 & 0 & 1
\end{array}\right),
$$

that $b=0$ for all $g \in G$.

It is not hard to check that if $g \in G$, and $v \in V$, then $a v \in V$ (just conjugate by $g$ ). Lemma 5.3 implies that there exists $n_{2} \in \mathbb{N}$ such that $a^{n_{2}}=1$ for every $g \in G$.

Case 5. $B=0, W \neq 0$ and $V=0$.

In this case, $W$ is an abelian subgroup of $\mathbb{C}$ and it is not hard to check that there exists $n_{1} \in \mathbb{N}$ such that $d^{n_{1}}=1$ for every $g \in G$. Now we consider the normal subgroup $N_{1}$ consisting of all those elements in $G$ of the form

$$
\left(\begin{array}{ccc}
a & b & v \\
0 & 1 & w \\
0 & 0 & 1
\end{array}\right) .
$$

It is easy to check by straightforward computation that the commutator of any two elements in $G$ has the form

$$
\left(\begin{array}{lll}
1 & \beta & v \\
0 & 1 & 0 \\
0 & 0 & 1
\end{array}\right)
$$

Since $B=V=0$, it follows that $N_{1}$ is an abelian group.

We can assume, conjugating by a suitable matrix, that every element in $N_{1}$ has the form

$$
\left(\begin{array}{ccc}
a & 0 & 0 \\
0 & 1 & w \\
0 & 0 & 1
\end{array}\right)
$$

It follows that $N_{1}$ is a (finitely generated) finite-index abelian subgroup of $G$.

Case 6. $B=0, W=0$ and $V \neq 0$.

In this case, $V \neq 0$ is an abelian subgroup of $\mathbb{C}$ and there is a fixed natural number $n_{1} \in \mathbb{N}$ such that $a^{n_{1}}=1$ for every $g \in G$. 
Now, let us denote by $N_{2}$ the normal subgroup of $G$ consisting of those elements of the form

$$
\left(\begin{array}{lll}
1 & b & v \\
0 & d & w \\
0 & 0 & 1
\end{array}\right)
$$

Let us fix an element

$$
h_{0}=\left(\begin{array}{ccc}
1 & b_{0} & v_{0} \\
0 & d_{0} & w_{0} \\
0 & 0 & 1
\end{array}\right) \in N_{2}, \quad d_{0} \neq 1,
$$

(if $d=1$ for every element in $N_{2}$, then the proof ends). If

$$
h=\left(\begin{array}{ccc}
1 & b & v \\
0 & d & w \\
0 & 0 & 1
\end{array}\right) \in N_{2},
$$

then $\left[h, h_{0}\right] \in N$. Hence

$$
(1-d) b_{0}+\left(1-d_{0}\right) b=0=(1-d) w_{0}+\left(1-d_{0}\right) w .
$$

Thus, conjugating by

$$
\left(\begin{array}{ccc}
1 & b_{0} /\left(1-d_{0}\right) & 0 \\
0 & d_{0}-1 & w_{0} \\
0 & 0 & 1
\end{array}\right),
$$

we can assume that every element in $N_{2}$ has the form

$$
h=\left(\begin{array}{lll}
1 & 0 & v \\
0 & d & 0 \\
0 & 0 & 1
\end{array}\right) .
$$

It follows that $N_{2}$ is a (finitely generated) finite-index abelian subgroup of $G$.

Case 7. $B=0, W=0$ and $V=0$.

In this case, every element in $G$ is of finite order, so $G$ is finite.

\section{Proof of Theorem 1.1(iii).}

If $G$ contains the ellipto-parabolic element $E_{1}$ then Proposition 5.6 implies the group $N_{1}$ is a finite-index normal abelian subgroup, and no element in $N_{1}$ acts on $\ell$ as a parabolic element. If we assume that there exists $g \in G$ such that $\phi(g)$ acts on $\ell$ as a parabolic element then for some $n_{0} \in \mathbb{N}$, we have that $\phi(g)^{n_{0}} \in \phi\left(N_{1}\right)$ acts as a parabolic element on $\ell$, which is a contradiction. A similar argument, using Proposition 5.6, shows that $G$ cannot contain the ellipto-parabolic element $E_{2}$.

The second part is analogous to the proof of Theorem 4.1 in [Fillmore and Scheuneman 1973], and we include it here for reader's convenience. By Proposition 5.8, 
$G$ is a finite extension of a discrete unipotent group $N$, and by Theorem 5.4.3 in [Corwin and Greenleaf 1990], there exists a unique Lie unipotent group $H$ such that $H / N$ is compact (in consequence, $N$ is finitely generated). This group $H$ is necessarily simply connected, and in fact it is a Euclidean space.

Since $N$ acts properly discontinuously (and freely) on $\mathbb{C}^{2}$, it follows that the projective dimension of the integer group ring of $N$ is less or equal to four (see [Cartan and Eilenberg 1999]). Moreover, the dimension of $H$ is less or equal to four.

The nilpotent simply connected Lie groups of dimension four are $\mathbb{R}^{4}$ and $H_{3} \times \mathbb{R}$, where $H_{3}$ denotes the real Heisenberg group. The nilpotent simply connected Lie groups of dimension three are $H_{3}$ and $\mathbb{R}^{3}$. Finally, those nilpotent simply connected Lie groups of dimension two and one are $\mathbb{R}^{2}$ and $\mathbb{R}$.

The discrete subgroups with compact quotient of $H_{3} \times \mathbb{R}$ are of the form

$$
\Delta_{k}=\left\langle A, B, C, D: C, D \text { are central and }[A, B]=C^{k}\right\rangle,
$$

where $k \in \mathbb{N}$. The discrete subgroups of $H_{3}$ with compact quotient are of the form

$$
\Gamma_{k}=\left\langle A, B, C: C \text { is central and }[A, B]=C^{k}\right\rangle,
$$

where $k \in \mathbb{N}$.

Acknowledgements. We would like to thank Professor John Parker for enlightening discussions during the process of this work and the kind hospitality received by the three authors at Durham University. Also, we would like to thank the referee for his helpful suggestions.

\section{References}

[Barrera Vargas et al. 2011] W. d. J. Barrera Vargas, A. C. Cordero, and J. P. N. Carrillo, "The limit set of discrete subgroups of PSL(3, C)", Math. Proc. Cambridge Philos. Soc. 150:1 (2011), 129-146. MR 2012b:32037 Zbl 1214.30032

[Cano and Seade 2010] A. Cano and J. Seade, "On the equicontinuity region of discrete subgroups of PU(1,n)”, J. Geom. Anal. 20:2 (2010), 291-305. MR 2011c:32045 Zbl 1218.37059

[Cano and Seade 2014] A. Cano and J. Seade, "On discrete groups of automorphisms of $\mathbb{P}_{\mathbb{C}}^{2}$ ", Geom. Dedicata 168 (2014), 9-60. MR 3158029 Zbl 06269858

[Cano et al. 2013] A. Cano, J. P. Navarrete, and J. Seade, Complex Kleinian groups, Progress in Mathematics 303, Birkhäuser, Basel, 2013. MR 2985759 Zbl 1267.30001

[Cartan and Eilenberg 1999] H. Cartan and S. Eilenberg, Homological algebra, Princeton University Press, 1999. MR 2000h:18022 Zbl 0933.18001

[Corwin and Greenleaf 1990] L. J. Corwin and F. P. Greenleaf, Representations of nilpotent Lie groups and their applications, I: Basic theory and examples, Cambridge Studies in Advanced Mathematics 18, Cambridge University Press, 1990. MR 92b:22007 Zbl 0704.22007

[Dekimpe 1996] K. Dekimpe, Almost-Bieberbach groups: Affine and polynomial structures, Lecture Notes in Mathematics 1639, Springer, Berlin, 1996. MR 2000b:20066 Zbl 0865.20001 
[Fillmore and Scheuneman 1973] J. P. Fillmore and J. Scheuneman, "Fundamental groups of compact complete locally affine complex surfaces”, Pacific J. Math. 44 (1973), 487-496. MR 48 \#6474 Zbl 0259.32008

[Kulkarni 1978] R. S. Kulkarni, "Groups with domains of discontinuity”, Math. Ann. 237:3 (1978), 253-272. MR 81m:30046 Zbl 0369.20028

[Marden 2007] A. Marden, Outer circles: An introduction to hyperbolic 3-manifolds, Cambridge University Press, 2007. MR 2008i:57001 Zbl 1149.57030

[Maskit 1988] B. Maskit, Kleinian groups, Grundlehren der Mathematischen Wissenschaften 287, Springer, Berlin, 1988. MR 90a:30132 Zbl 0627.30039

[Navarrete 2008] J.-P. Navarrete, "The trace function and complex Kleinian groups in $\mathbb{P}_{\mathbb{C}}^{2}$ ", Internat. J. Math. 19:7 (2008), 865-890. MR 2009g:32056 Zbl 1167.30025

[Ratcliffe 1994] J. G. Ratcliffe, Foundations of hyperbolic manifolds, Graduate Texts in Mathematics 149, Springer, New York, 1994. MR 95j:57011 Zbl 0809.51001

[Scheuneman 1974] J. Scheuneman, "Fundamental groups of compact complete locally affine complex surfaces, II”, Pacific J. Math. 52 (1974), 553-566. MR 54 \#612 Zbl 0291.32029

[Suwa 1975] T. Suwa, "Compact quotient spaces of $\mathbf{C}^{2}$ by affine transformation groups", J. Differential Geometry 10 (1975), 239-252. MR 51 \#5977 Zbl 0311.57025

Received September 26, 2013. Revised December 17, 2013.

WALDEMAR BARRERA

FACULTAD DE MATEMÁticas

UNIVERSIDAD AUTÓNOMA DE YUCATÁN

ANillo Periférico Norte Tablaje CAT

13615 MÉRIDA, YUCATÁN

MÉXICO

bvargas@uady.mx

ANGEL CANO

InSTITUTO DE MATEMÁticas

Universidad NaCional Autónoma de MÉXICO

AV. UNIVERSIDAD S/N

Col. LOMAS DE CHAMilpa

62210 CuernavaCA, Morelos

MÉXICO

angel@matcuer.unam.mx

JuAN PABlo NAVARRETE

FACULTAD DE MATEMÁticas

UNIVERSIDAD AUTÓNOMA DE YUCATÁN

Anillo Periférico Norte Tablaje CAT

13615 MÉRIDA, YUCATÁN

MÉXICO

jp.navarrete@uady.mx 


\title{
PACIFIC JOURNAL OF MATHEMATICS
}

\author{
msp.org/pjm
}

Founded in 1951 by E. F. Beckenbach (1906-1982) and F. Wolf (1904-1989)

\section{EDITORS}

Don Blasius (Managing Editor)

Department of Mathematics

University of California

Los Angeles, CA 90095-1555

blasius@math.ucla.edu

\author{
Paul Balmer \\ Department of Mathematics \\ University of California \\ Los Angeles, CA 90095-1555 \\ balmer@math.ucla.edu \\ Robert Finn \\ Department of Mathematics \\ Stanford University \\ Stanford, CA 94305-2125 \\ finn@math.stanford.edu \\ Sorin Popa \\ Department of Mathematics \\ University of California \\ Los Angeles, CA 90095-1555 \\ popa@math.ucla.edu
}

\author{
Vyjayanthi Chari \\ Department of Mathematics \\ University of California \\ Riverside, CA 92521-0135 \\ chari@math.ucr.edu \\ Kefeng Liu \\ Department of Mathematics \\ University of California \\ Los Angeles, CA 90095-1555 \\ liu@math.ucla.edu \\ Jie Qing \\ Department of Mathematics \\ University of California \\ Santa Cruz, CA 95064 \\ qing@ cats.ucsc.edu
}

\section{PRODUCTION}

Silvio Levy, Scientific Editor, production@msp.org

\section{SUPPORTING INSTITUTIONS}

ACADEMIA SINICA, TAIPEI

CALIFORNIA INST. OF TECHNOLOGY

INST. DE MATEMÁTICA PURA E APLICADA

KEIO UNIVERSITY

MATH. SCIENCES RESEARCH INSTITUTE

NEW MEXICO STATE UNIV.

OREGON STATE UNIV.

\author{
STANFORD UNIVERSITY \\ UNIV. OF BRITISH COLUMBIA \\ UNIV. OF CALIFORNIA, BERKELEY \\ UNIV. OF CALIFORNIA, DAVIS \\ UNIV. OF CALIFORNIA, LOS ANGELES \\ UNIV. OF CALIFORNIA, RIVERSIDE \\ UNIV. OF CALIFORNIA, SAN DIEGO \\ UNIV. OF CALIF., SANTA BARBARA
}

\author{
Daryl Cooper \\ Department of Mathematics \\ University of California \\ Santa Barbara, CA 93106-3080 \\ cooper@math.ucsb.edu \\ Jiang-Hua Lu \\ Department of Mathematics \\ The University of Hong Kong \\ Pokfulam Rd., Hong Kong \\ jhlu@maths.hku.hk \\ Paul Yang \\ Department of Mathematics \\ Princeton University \\ Princeton NJ 08544-1000 \\ yang@math.princeton.edu
}

These supporting institutions contribute to the cost of publication of this Journal, but they are not owners or publishers and have no responsibility for its contents or policies.

See inside back cover or msp.org/pjm for submission instructions.

The subscription price for 2014 is US $\$ 410 /$ year for the electronic version, and \$535/year for print and electronic.

Subscriptions, requests for back issues and changes of subscribers address should be sent to Pacific Journal of Mathematics, P.O. Box 4163, Berkeley, CA 94704-0163, U.S.A. The Pacific Journal of Mathematics is indexed by Mathematical Reviews, Zentralblatt MATH, PASCAL CNRS Index, Referativnyi Zhurnal, Current Mathematical Publications and Web of Knowledge (Science Citation Index).

The Pacific Journal of Mathematics (ISSN 0030-8730) at the University of California, c/o Department of Mathematics, 798 Evans Hall \#3840, Berkeley, CA 94720-3840, is published twelve times a year. Periodical rate postage paid at Berkeley, CA 94704, and additional mailing offices. POSTMASTER: send address changes to Pacific Journal of Mathematics, P.O. Box 4163, Berkeley, CA 94704-0163.

PJM peer review and production are managed by EditFLOW ${ }^{\circledR}$ from Mathematical Sciences Publishers.

\section{PUBLISHED BY}

\section{mathematical sciences publishers \\ nonprofit scientific publishing}

http://msp.org/

(C) 2014 Mathematical Sciences Publishers 


\section{PACIFIC JOURNAL OF MATHEMATICS}

Volume $272 \quad$ No. $2 \quad$ December 2014

Marginally trapped submanifolds in space forms with arbitrary

signature

HENRI ANCIAUX

One line complex Kleinian groups

275

Waldemar Barrera, Angel Cano and JuAn Pablo

NAVARRETE

A note on flux integrals over smooth regular domains

305

IDO BRIGHT and JOHN M. LEE

On stable commutator length in hyperelliptic mapping class groups

323

DANny Calegari, NaOyuKi Monden and Masatoshi Sato

Compositions of birational endomorphisms of the affine plane

PierRetTe CASSOU-NoguÈs and DANIEl DAigle

The Bochner formula for isometric immersions

395

ALESSANDRO SAVO

On solutions to Cournot-Nash equilibria equations on the sphere

423

MICAH WARREN

Double and triple Givental's J-functions for stable quotients invariants

439

ALEKSEY ZiNGER

Acknowledgement 